Supporting Information

\title{
Proton Coupled Electron Transfer in a Strongly Coupled Photosystem II-Inspired Chromophore-Imidazole-Phenol Complex: Stepwise Oxidation and Concerted Reduction
}

\author{
Gerald F. Manbeck*, Etsuko Fujita, and Javier J. Concepcion* \\ Chemistry Division, Brookhaven National Laboratory, Upton, NY 11973-5000, United States. \\ *Email: gmanbeck@bnl.gov, jconcepc@bnl.gov
}

\section{Contents:}

Figures S1-15

NMR spectra

S2-S9

Figure $\mathrm{S} 16$

Infrared spectra $(\mathrm{KBr})$

S10

Figure S17

UV-vis absorption spectra

S10

Emission spectra $\quad$ S11

Table S1

Photophysical data

S11

Figure S19

Stern-Volmer quenching

S12

Excited state transient absorption spectra $\quad$ S13

Figure S20

Transient absorption spectra and kinetic traces with $5 \mathrm{mM}$ methyl

S14

Figure S22 viologen

Temperature dependence of the phenoxyl radical reduction by the methyl $\mathbf{S 1 5}$

Table S2

Table S3

Figures S23-27 viologen radical cation.

Kinetic data for intramolecular phenol oxidation

S16

Kinetic data for phenoxyl radical reduction by $\mathrm{MV}^{\circ+} \quad \mathbf{S 1 6}$

Cyclic voltammetry

S17-19

Summary of electrochemical data

S20

Table S5

Calculated $\mathrm{OH}$ and $\mathrm{NH}^{+}$IR stretching frequencies

S21

Figure S28

Spin density plots of Ru(III) and phenoxyl radicals before and after PCET

S21

Tables S6-9

Cartesian coordinates of DFT structures

S21-31

References 
बN.

25 0 \%

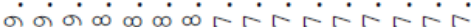

N
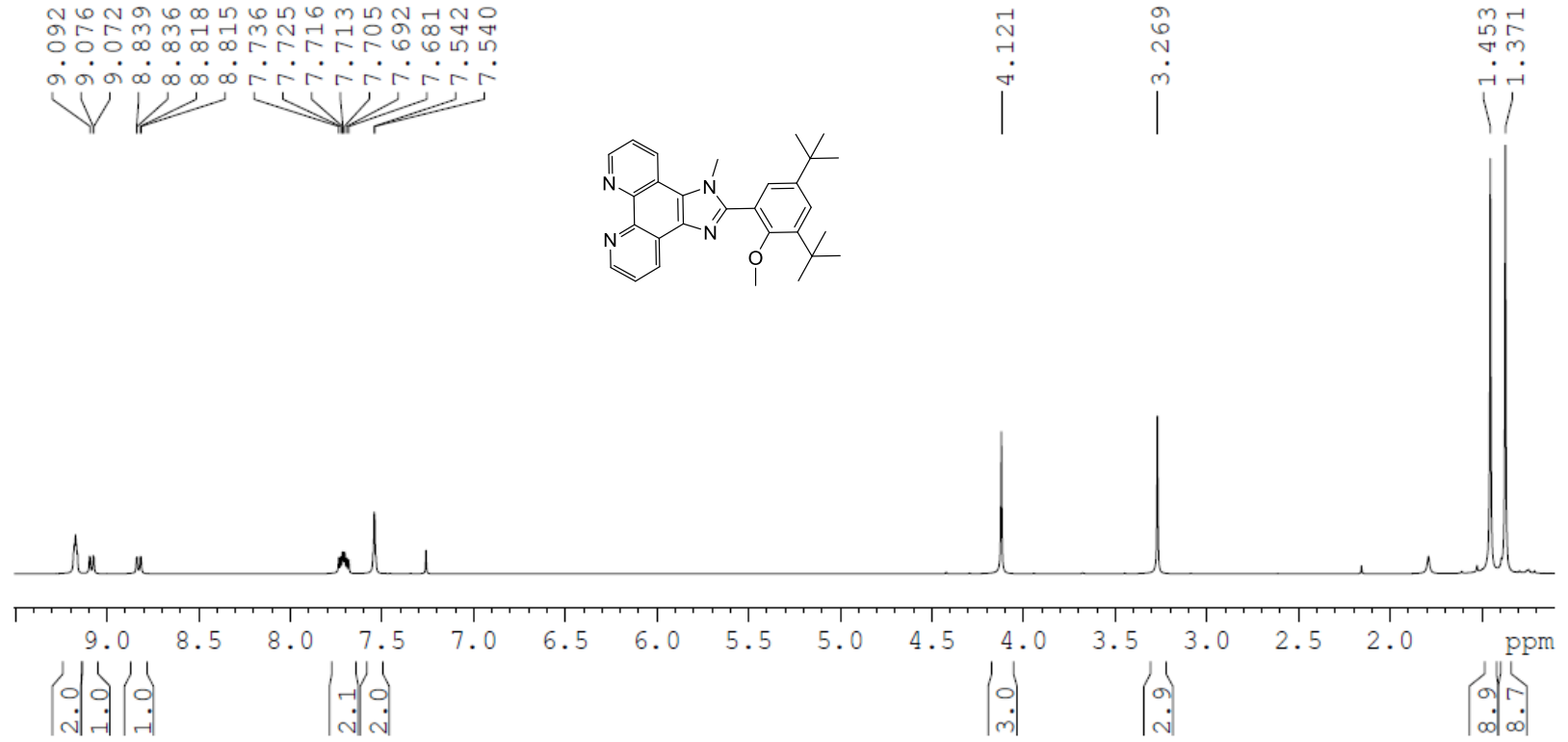

Figure S1. ${ }^{1} \mathrm{H}$ NMR spectrum of (phen-Im-PhOMe) in $\mathrm{CDCl}_{3}$.

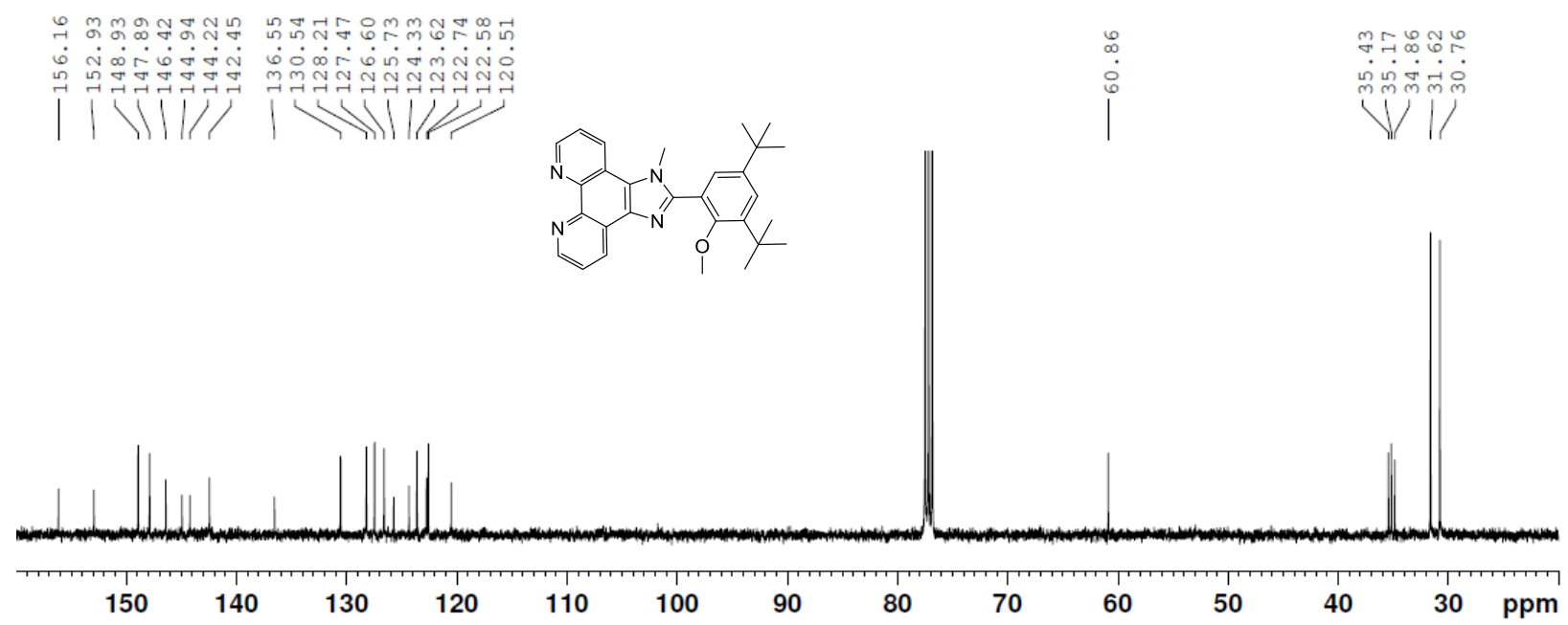

Figure S2. ${ }^{13} \mathrm{C}$ NMR spectrum of (phen-Im-PhOMe) in $\mathrm{CDCl}_{3}$. 

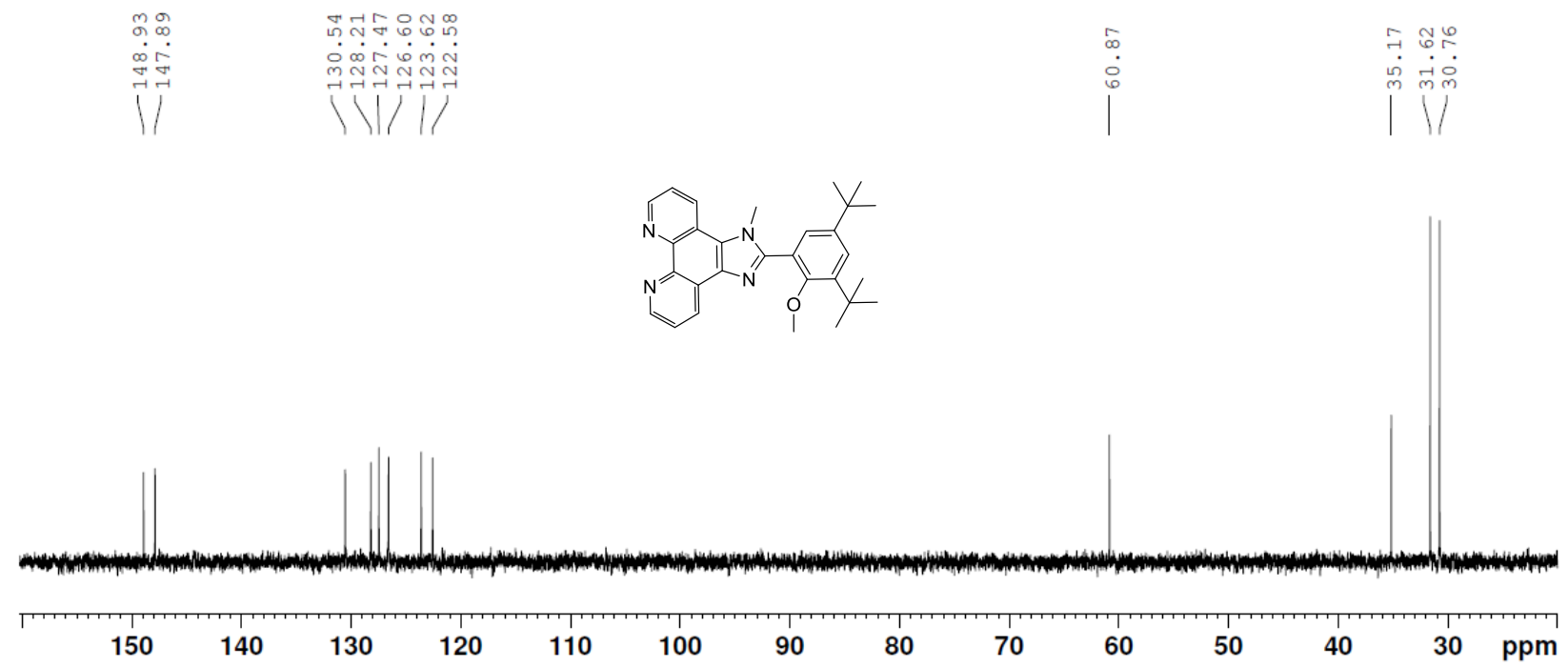

Figure S3. ${ }^{13} \mathrm{C}$ DEPT NMR spectrum of (phen-Im-PhOMe) in $\mathrm{CDCl}_{3}$.
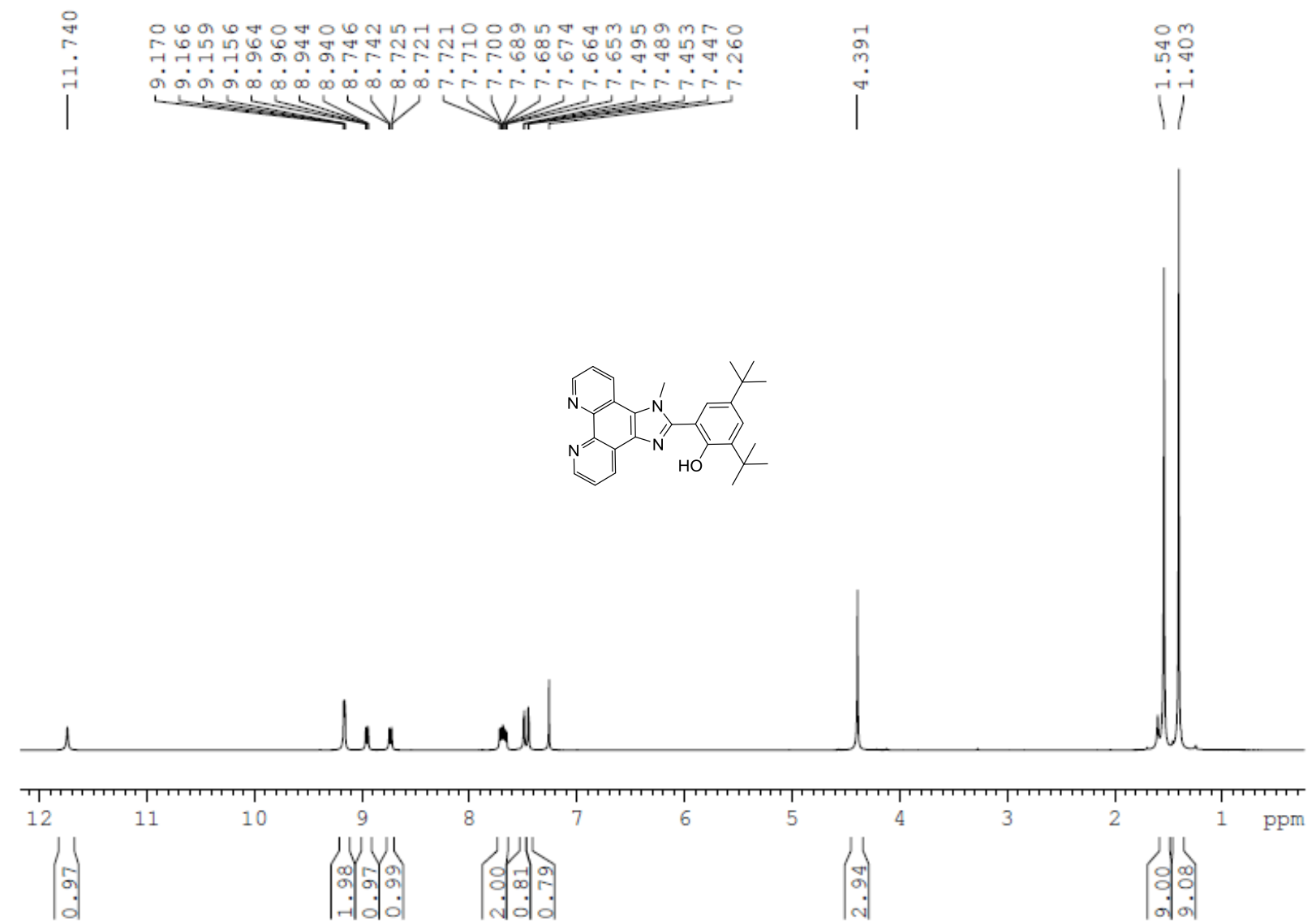

Figure S4. ${ }^{1} \mathrm{H}$ NMR spectrum of (phen-Im-PhOH) in $\mathrm{CDCl}_{3}$. 


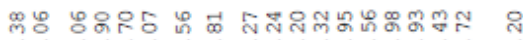

मिं

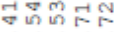

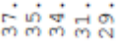

IIVIINMVI)

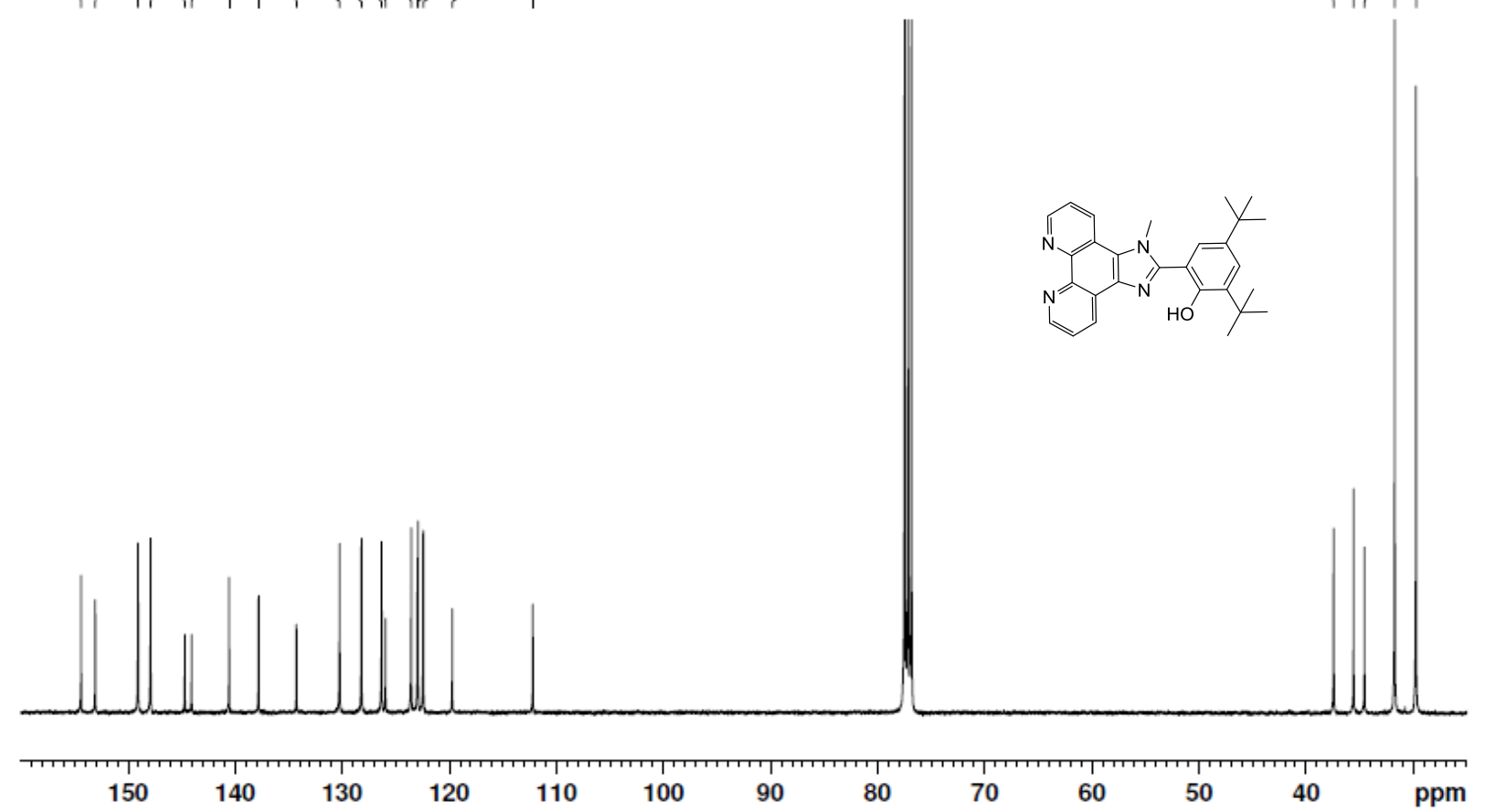

Figure S5. ${ }^{13} \mathrm{C}$ NMR spectrum of (phen-Im-PhOH) in $\mathrm{CDCl}_{3}$.
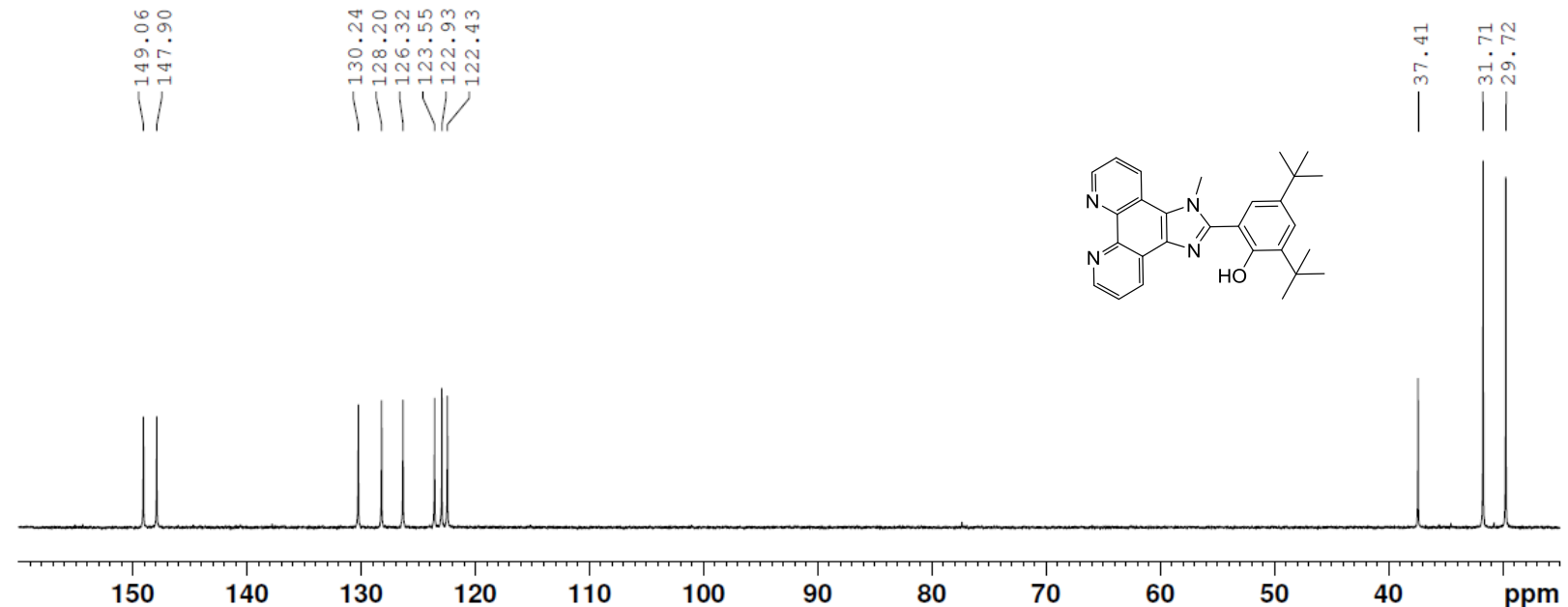

Figure S6. ${ }^{13} \mathrm{C}$ DEPT NMR spectrum of (phen-Im-PhOH) in $\mathrm{CDCl}_{3}$. 


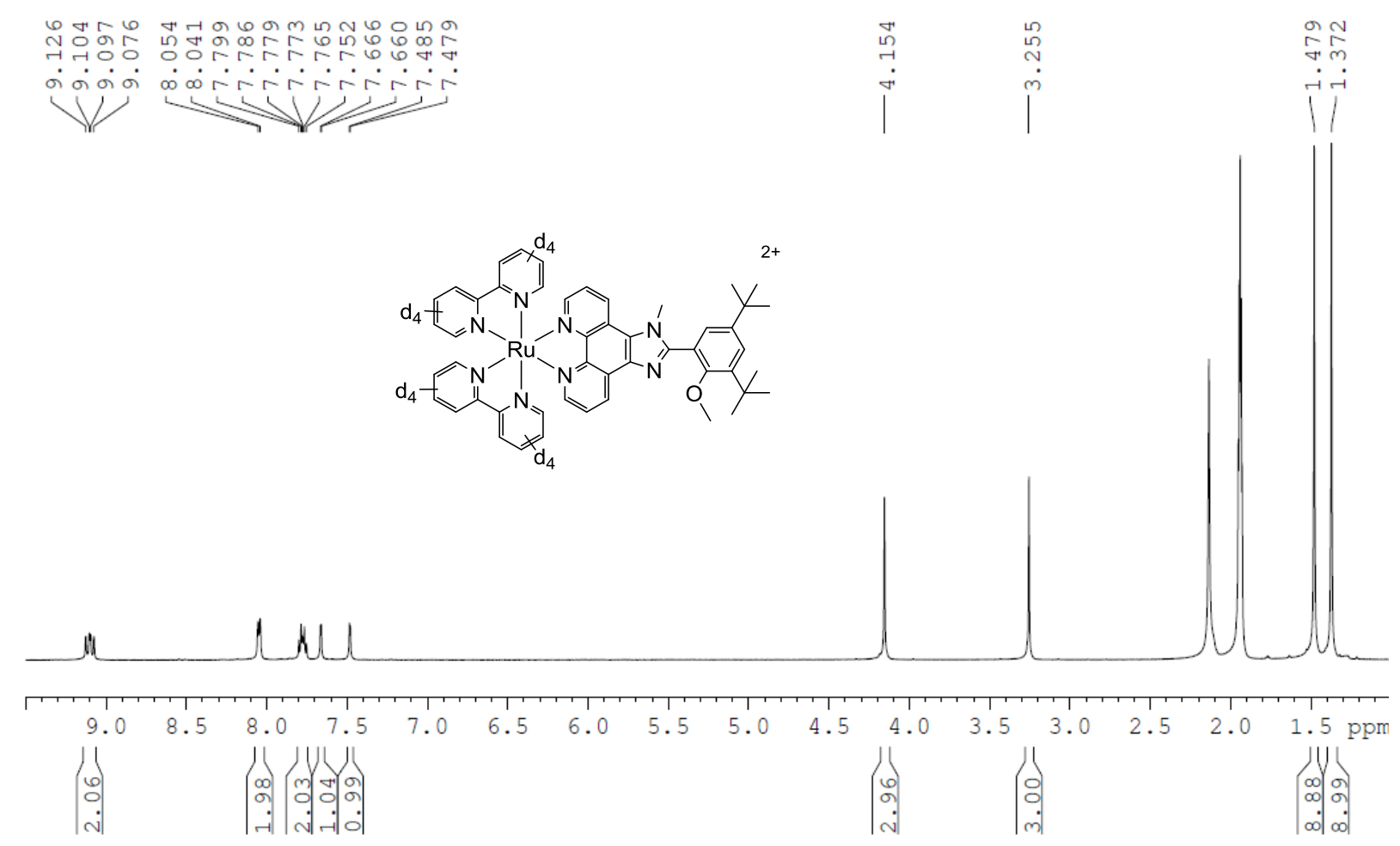

Figure S7. ${ }^{1} \mathrm{H}$ NMR of $\left[\left(\text { bpy- } d_{8}\right)_{2} \mathrm{Ru}(\right.$ phen-Im-PhOMe $\left.)\right]\left(\mathrm{PF}_{6}\right)_{2}$ in $\mathrm{CD}_{3} \mathrm{CN}$.

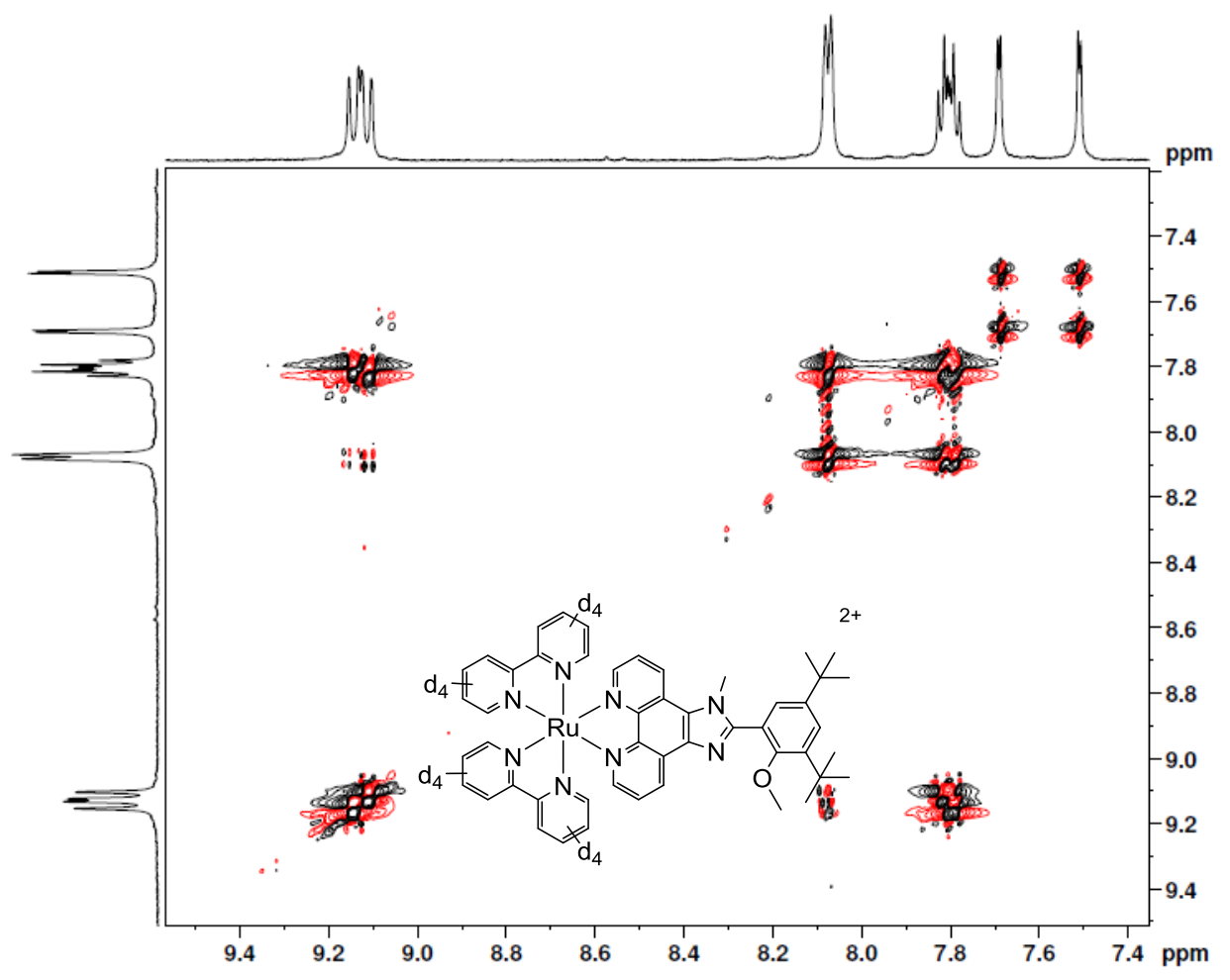

Figure S8. ${ }^{1} \mathrm{H}$ COSY NMR of $\left[\left(\text { bpy- } d_{8}\right)_{2} \mathrm{Ru}(\right.$ phen-Im-PhOMe $\left.)\right]\left(\mathrm{PF}_{6}\right)_{2}$ in $\mathrm{CD}_{3} \mathrm{CN}$. 


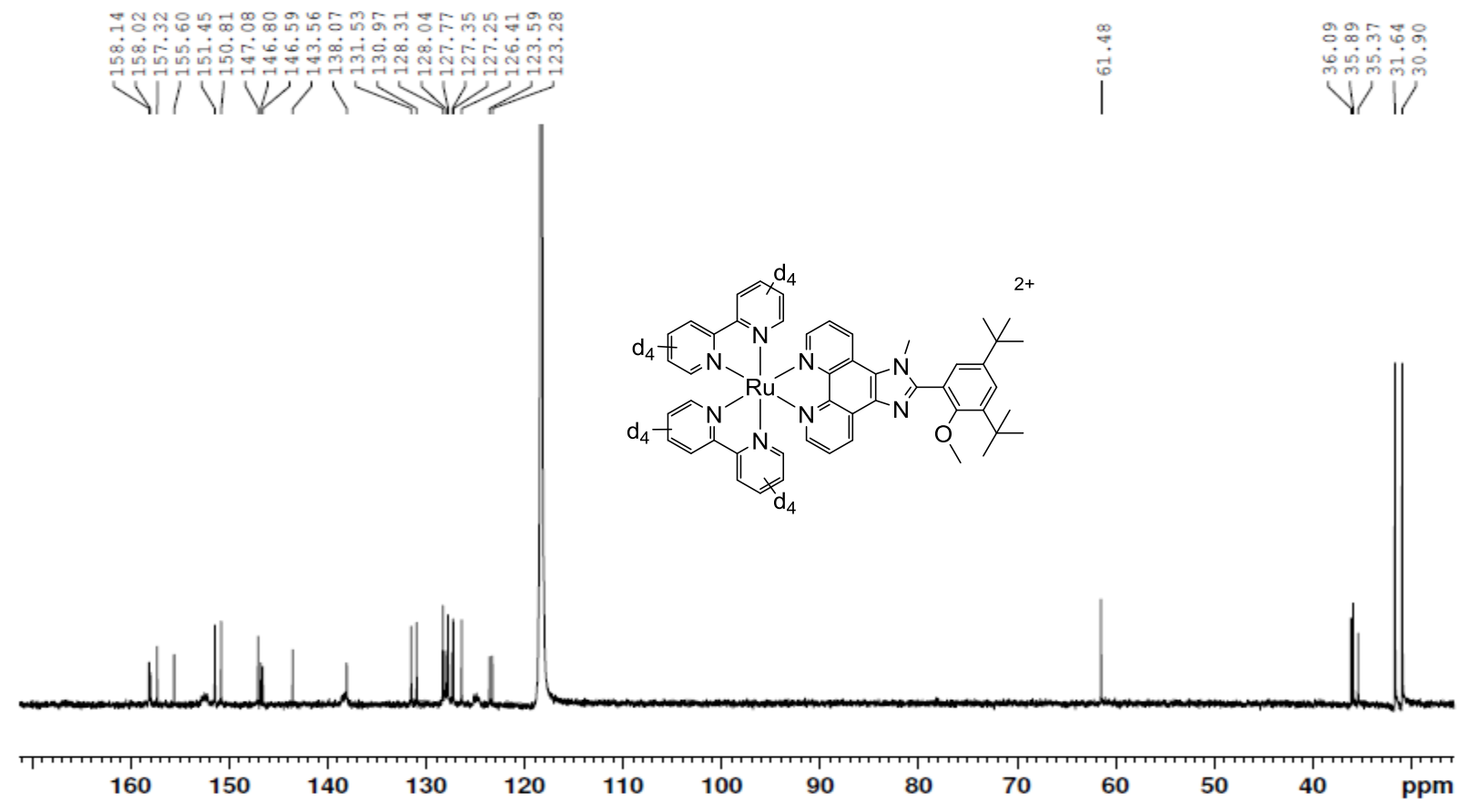

Figure S9. ${ }^{13} \mathrm{C}$ NMR of $\left[\left(\text { bpy- } d_{8}\right)_{2} \mathrm{Ru}(\right.$ phen-Im-PhOMe $\left.)\right]\left(\mathrm{PF}_{6}\right)_{2}$ in $\mathrm{CD}_{3} \mathrm{CN}$.
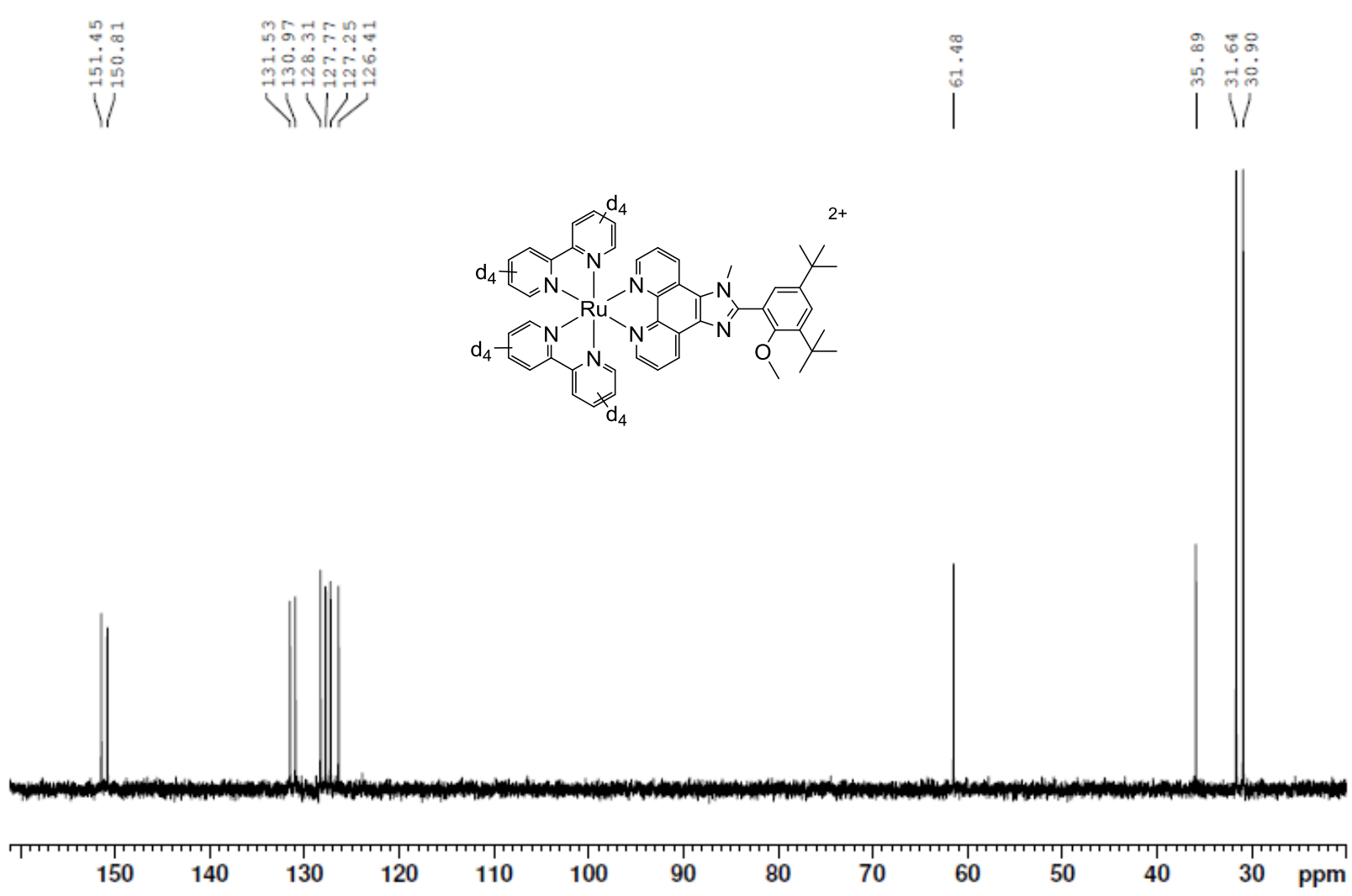

Figure S10. ${ }^{13} \mathrm{C}$ DEPT NMR of $\left[\left(\mathrm{bpy}-d_{8}\right)_{2} \mathrm{Ru}(\right.$ phen-Im-PhOMe $\left.)\right]\left(\mathrm{PF}_{6}\right)_{2}$ in $\mathrm{CD}_{3} \mathrm{CN}$. 


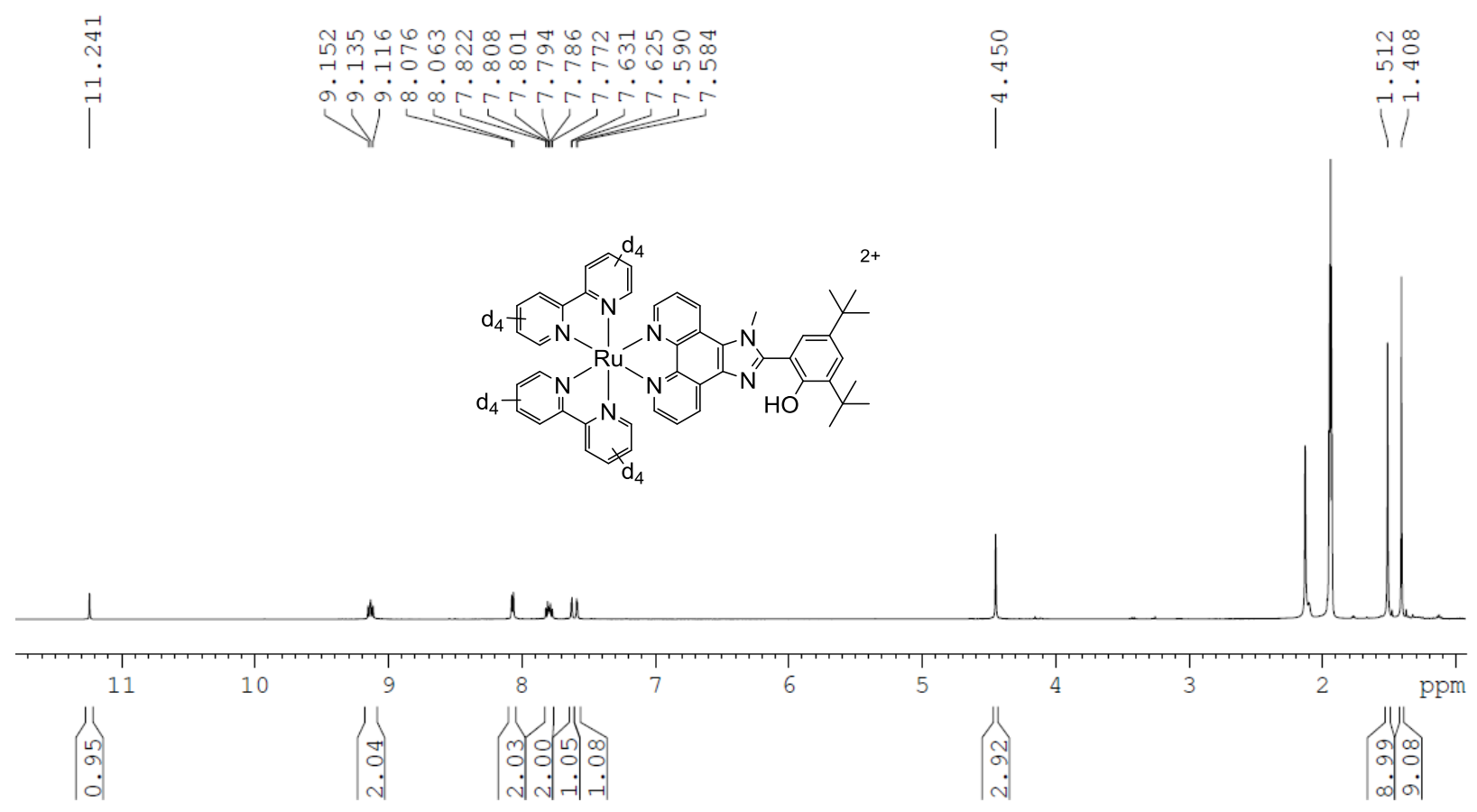

Figure S11. ${ }^{1} \mathrm{H} \mathrm{NMR}$ of $\left[\left(\mathrm{bpy}-d_{8}\right)_{2} \mathrm{Ru}(\right.$ phen-Im-PhOH$\left.)\right]\left(\mathrm{PF}_{6}\right)_{2}$ in $\mathrm{CD}_{3} \mathrm{CN}$.

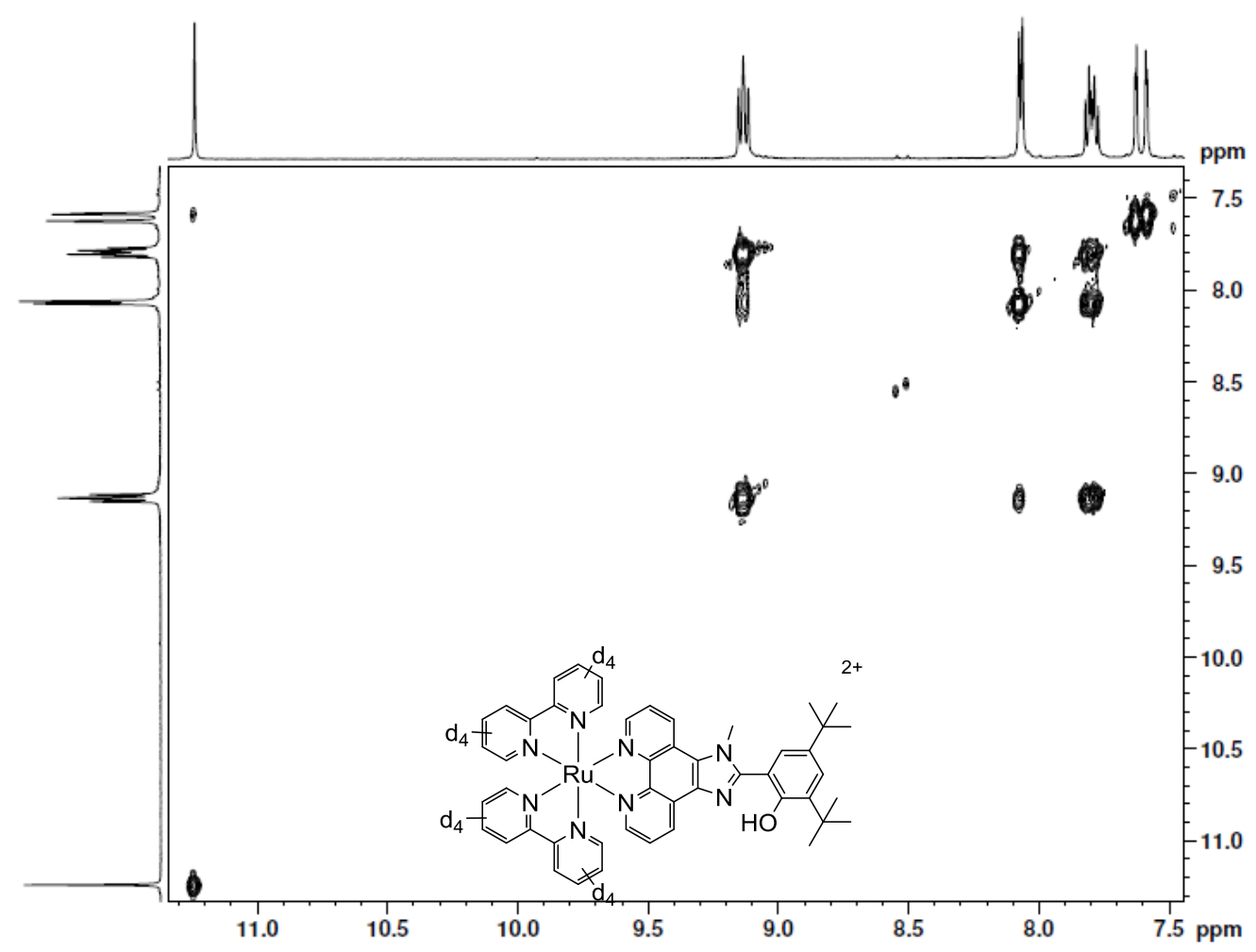

Figure S12. ${ }^{1} \mathrm{H}$ COSY NMR of $\left[\left(\mathrm{bpy}-d_{8}\right)_{2} \mathrm{Ru}(\mathrm{phen}-\mathrm{Im}-\mathrm{PhOH})\right]\left(\mathrm{PF}_{6}\right)_{2}$ in $\mathrm{CD}_{3} \mathrm{CN}$. 


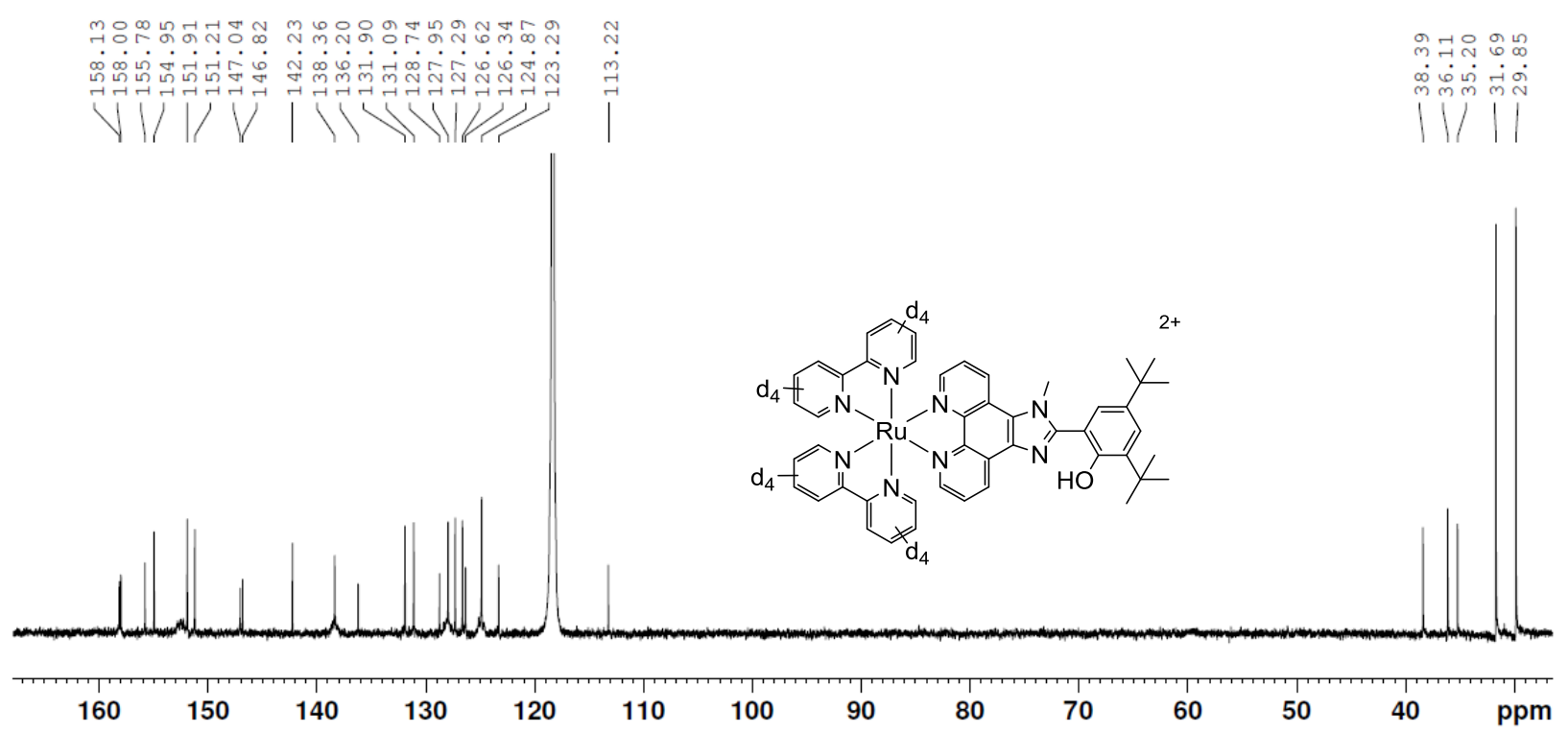

Figure S13. ${ }^{13} \mathrm{C}$ NMR of $\left[\left(\text { bpy- } d_{8}\right)_{2} \mathrm{Ru}(\right.$ phen-Im- $\left.\mathrm{PhOH})\right]\left(\mathrm{PF}_{6}\right)_{2}$ in $\mathrm{CD}_{3} \mathrm{CN}$.

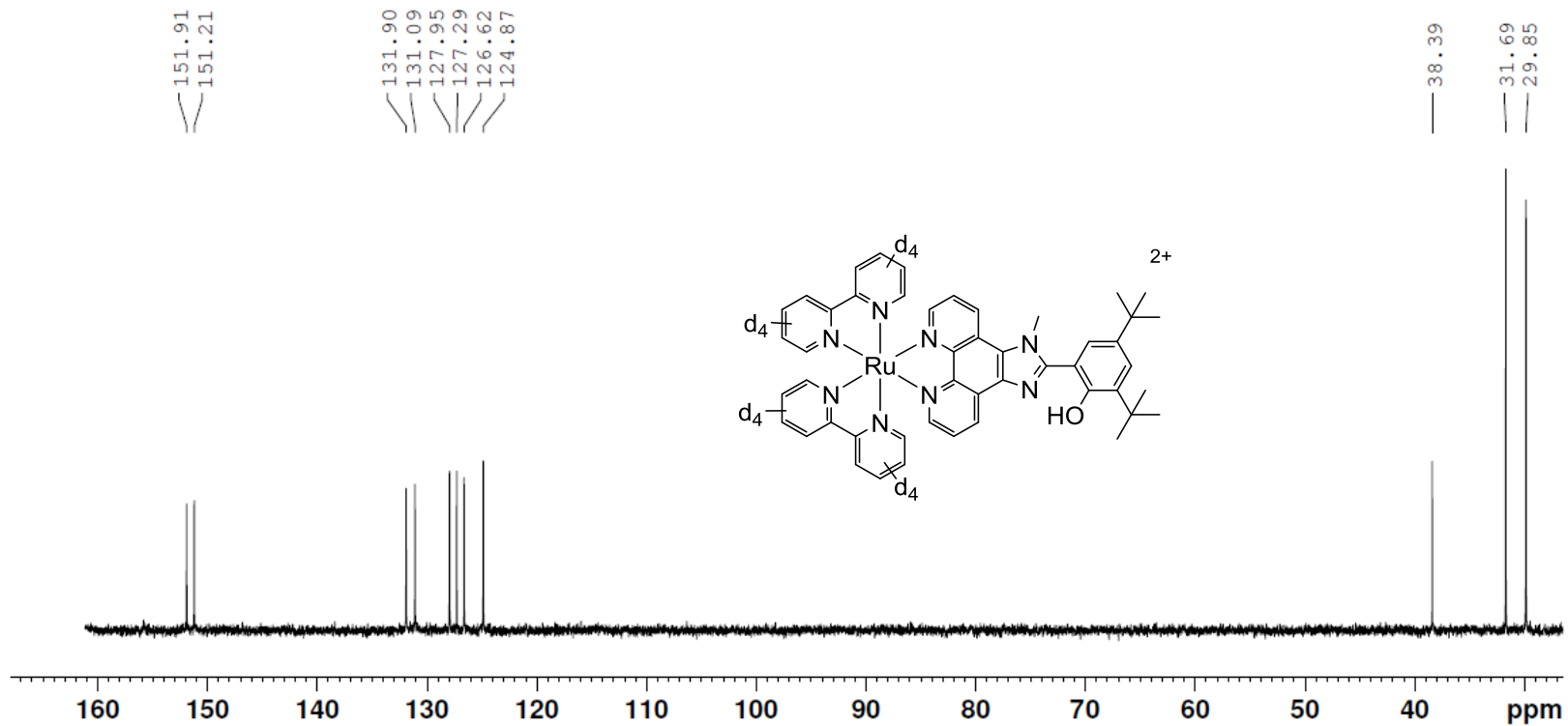

Figure S14. ${ }^{13} \mathrm{C}$ DEPT NMR of $\left[\left(\text { bpy- } d_{8}\right)_{2} \mathrm{Ru}(\right.$ phen-Im-PhOH $\left.)\right]\left(\mathrm{PF}_{6}\right)_{2}$ in $\mathrm{CD}_{3} \mathrm{CN}$. 


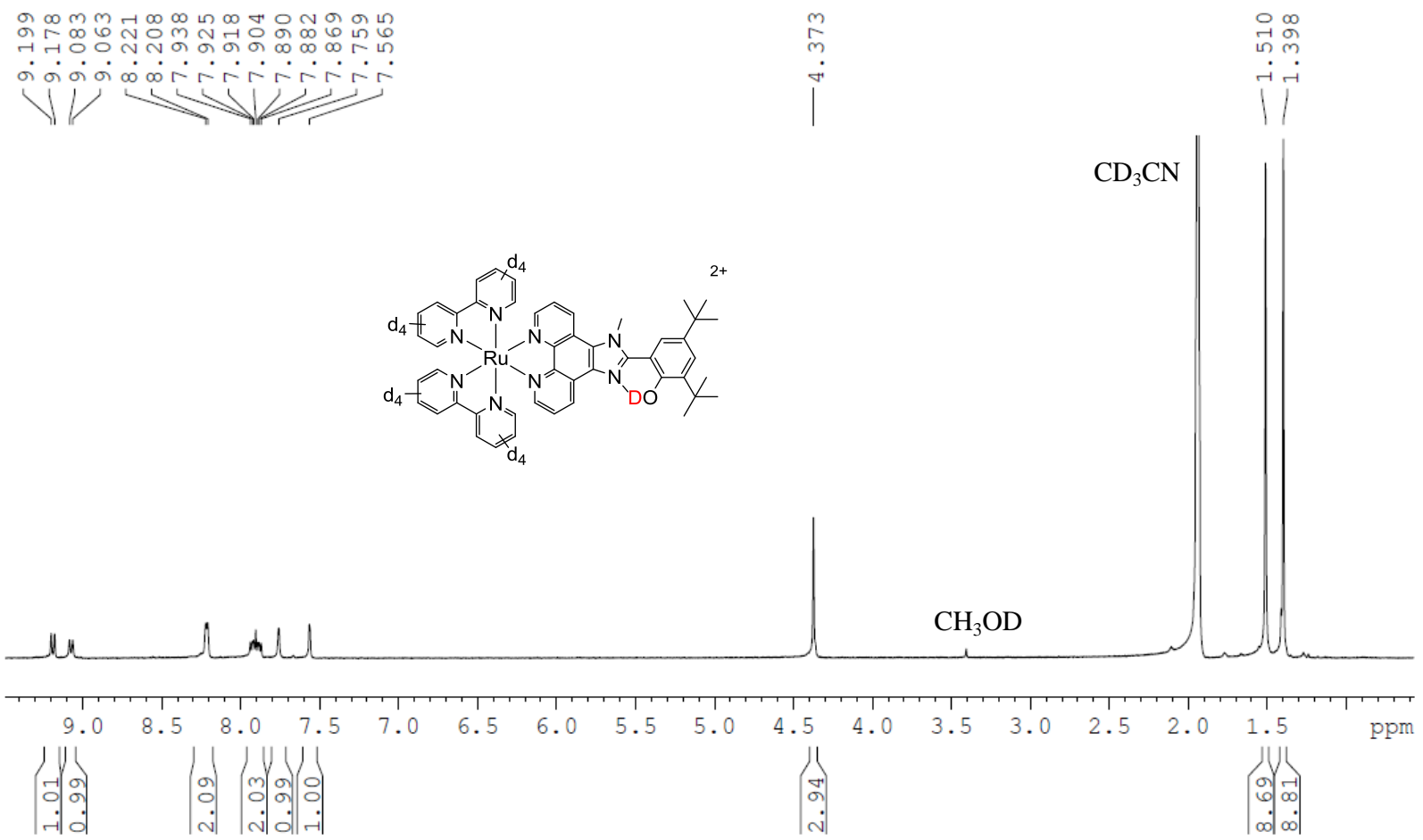

Figure S15. ${ }^{1} \mathrm{H}$ NMR spectrum of $\left[\left(\text { bpy- } d_{8}\right)_{2} \mathrm{Ru}(\mathrm{phen}-\mathrm{Im}-\mathrm{PhOD})\right]\left(\mathrm{PF}_{6}\right)_{2}$ in $\mathrm{CD}_{3} \mathrm{CN}$. 


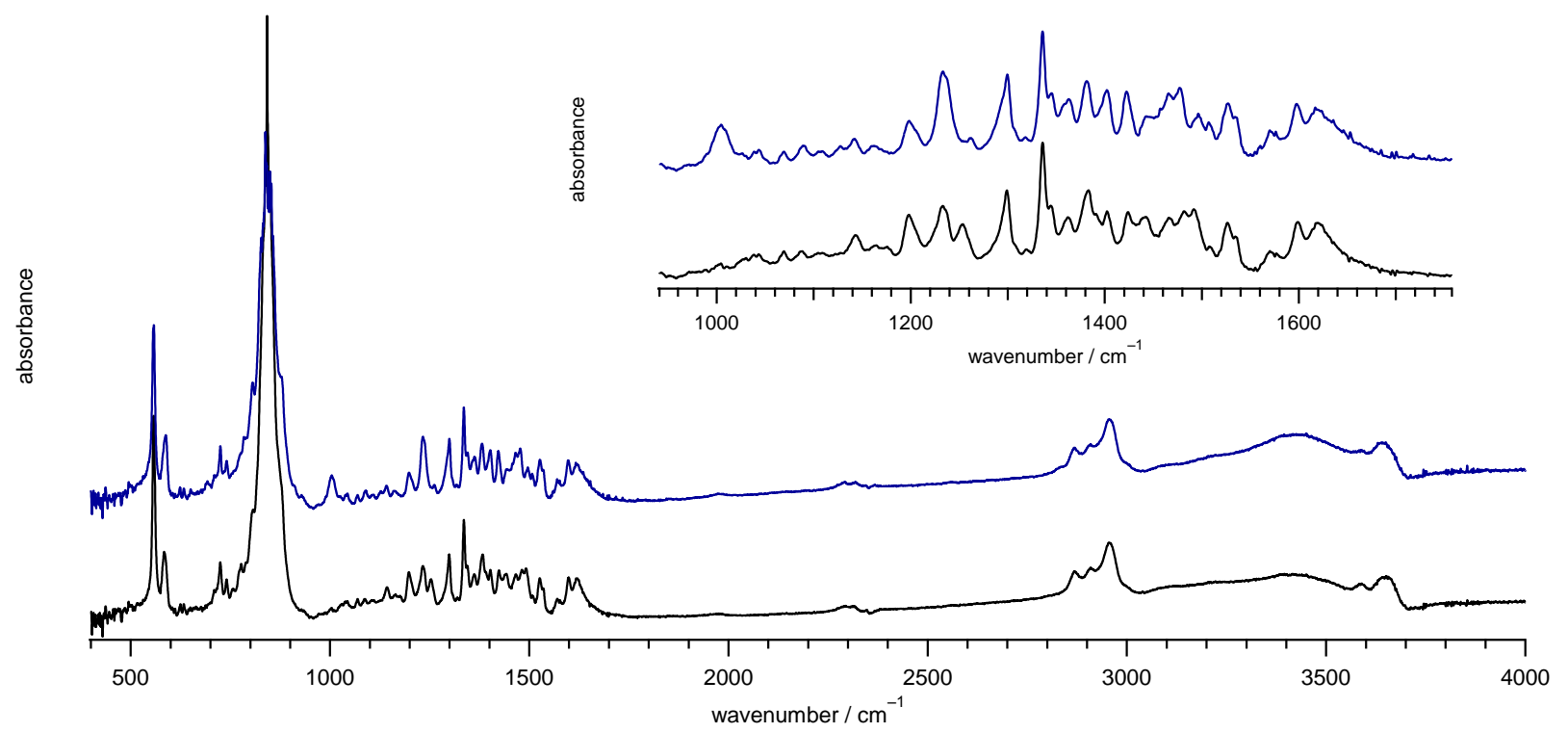

Figure S16. Infrared spectra of $\left[(\mathrm{bpy})_{2} \mathrm{Ru}^{\mathrm{II}}(\text { phen-Im-PhOH})\right]^{2+}$ (black, bottom) and $\left[(\mathrm{bpy})_{2} \mathrm{Ru}{ }^{\mathrm{II}}(\right.$ phen-Im$\mathrm{PhOMe}$ ) ${ }^{2+}$ (blue, top) in $\mathrm{KBr}$. Inset: expansion of the region 1000 to $1700 \mathrm{~cm}^{-1}$. The weak $\mathrm{OH}$ stretch is not evident due to its hydrogen bonded nature. Spectra of the $\mathrm{PhOH}$ and $\mathrm{PhOMe}$ complexes are virtually identical with the exception of the stretch at $1004 \mathrm{~cm}^{-1}$ which can be assigned as a CO stretch of the $\mathrm{PhO}-\mathrm{CH}_{3}$ by comparison to the analogous mode in anisole $\left(1039 \mathrm{~cm}^{-1}\right){ }^{1}$

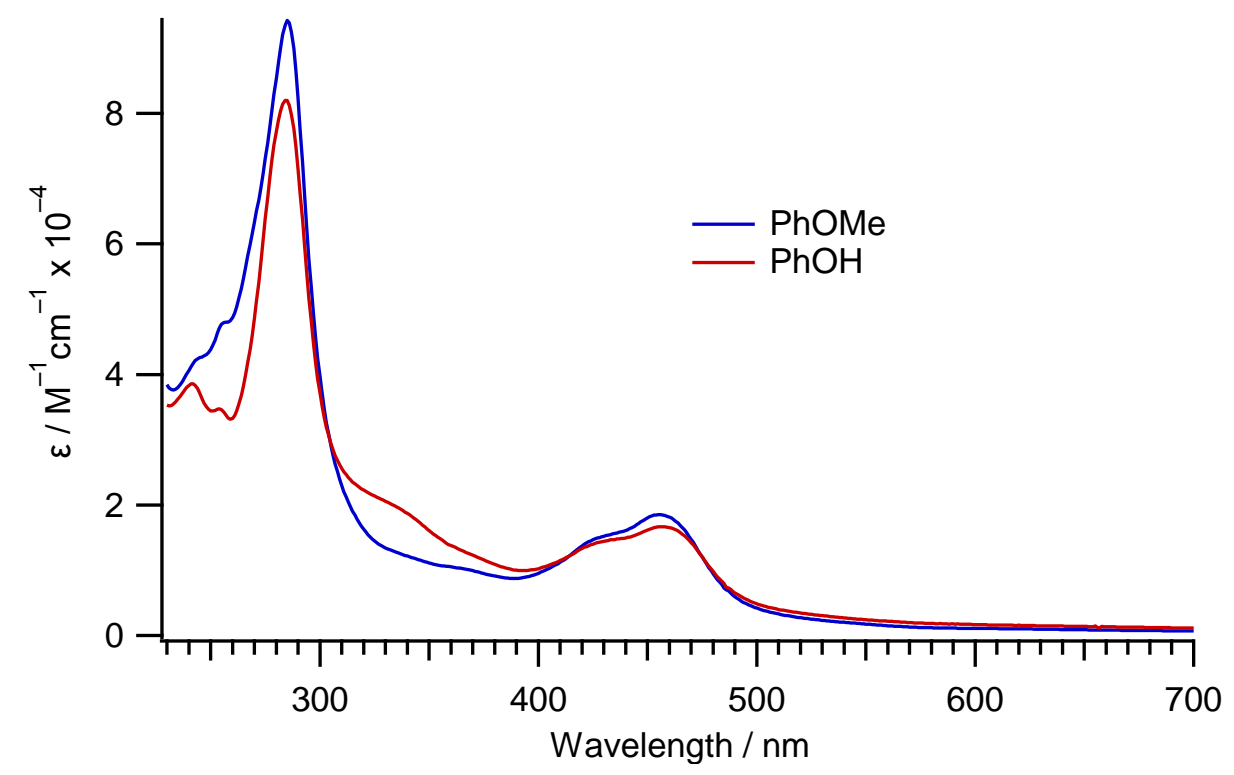

Figure S17. Absorption spectra of $\left[\left(\mathrm{bpy}-d_{8}\right)_{2} \mathrm{Ru}(\mathrm{phen}-\mathrm{Im}-\mathrm{PhOMe})\right]\left(\mathrm{PF}_{6}\right)_{2}$ and $\left[\left(\mathrm{bpy}-d_{8}\right)_{2} \mathrm{Ru}(\mathrm{phen}-\mathrm{Im}-\right.$ $\mathrm{PhOH})]\left(\mathrm{PF}_{6}\right)_{2}$ and in $\mathrm{CH}_{3} \mathrm{CN}$. 


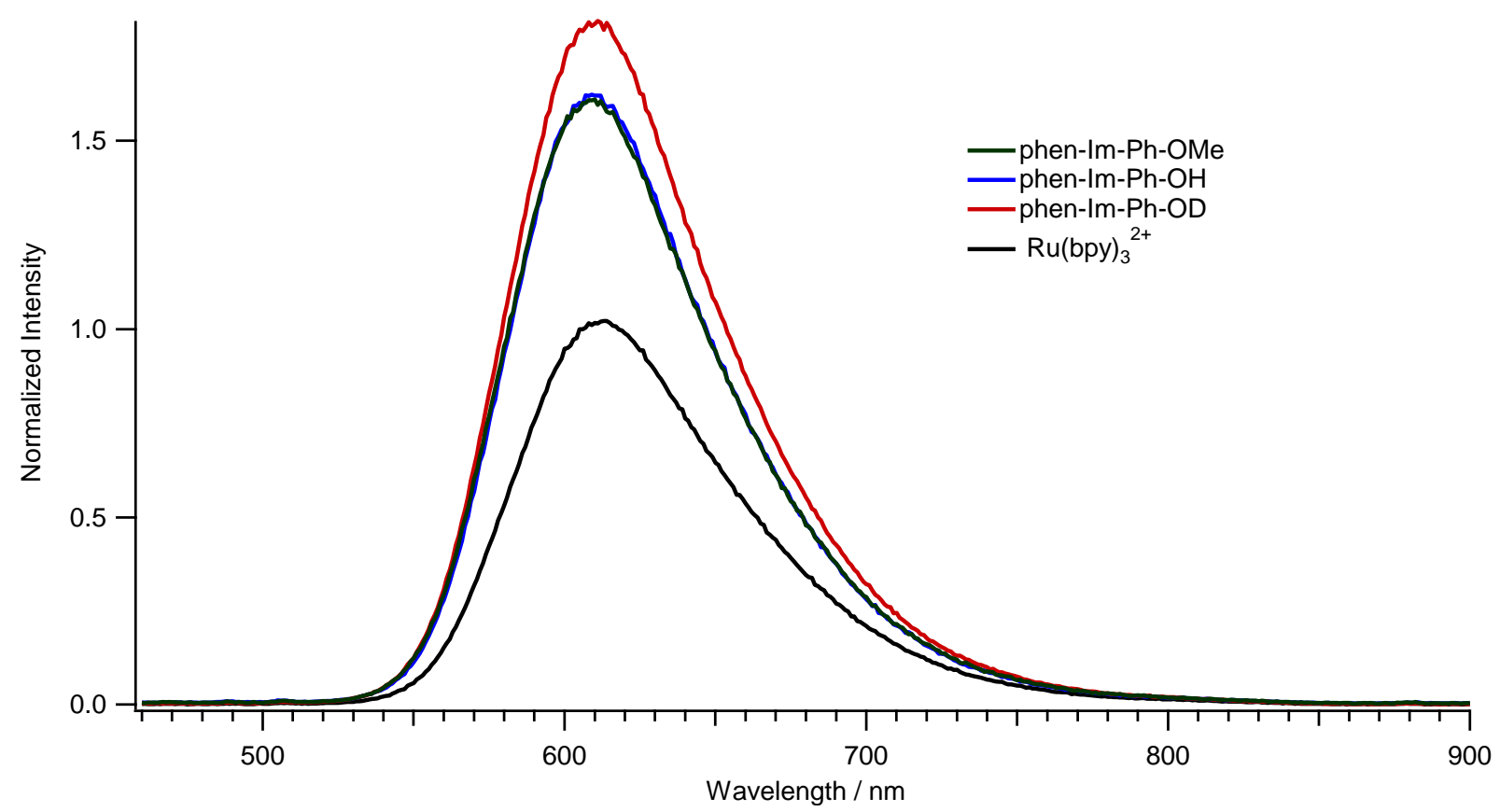

Figure S18. Steady state emission spectra of $\left[\left(\text { bpy- } d_{8}\right)_{2} \mathrm{Ru}(\right.$ phen-Im-PhOMe $\left.)\right]\left(\mathrm{PF}_{6}\right)_{2},\left[\left(\mathrm{bpy}-d_{8}\right)_{2} \mathrm{Ru}(\mathrm{phen}-\right.$ $\mathrm{Im}-\mathrm{PhOH})]\left(\mathrm{PF}_{6}\right)_{2},\left[\left(\mathrm{bpy}-d_{8}\right)_{2} \mathrm{Ru}(\right.$ phen-Im-PhOD $\left.)\right]\left(\mathrm{PF}_{6}\right)_{2}$, and $\left[\mathrm{Ru}(\mathrm{bpy})_{3}\right]^{2+}$ in deoxygenated $\mathrm{CH}_{3} \mathrm{CN}$. The intensities are normalized with respect to quantum yields of emission relative to $\left[\operatorname{Ru}(b p y)_{3}\right]^{2+}\left(\Phi_{\mathrm{em}}=\right.$ 0.062).

The $\mathrm{Ru}^{\mathrm{III} / \mathrm{II}}$ standard potential relative to both phenol couples indicates that $\mathrm{Ru}^{\mathrm{III}}$ can oxidize the $\mathrm{PhOH}$ via PCET or ET-PT pathways, given assignments of waves 1 and 2 (see text and Figure S24) as the $\mathrm{PhOH} / \mathrm{PhO}^{\circ}$ and $\mathrm{PhOH}^{+/ 0}$ couples. The absence of excited state intramolecular quenching as shown by the identical steady state emission intensities and lifetimes of Ru-phen-Im-PhOH/OMe are understood by analysis of the excited state $\mathrm{Ru}^{\mathrm{I}{ }^{* / I}}$ potential:

$$
E^{\mathrm{O}}\left(\mathrm{Ru}^{\mathrm{II} * / \mathrm{I}}\right)=E_{o o}+E^{\mathrm{O}}\left(\mathrm{Ru}^{\mathrm{II} / \mathrm{I}}\right)
$$

The emission energy of Ru-phen-Im- $\mathrm{PhOH}$ is identical to $\left[\mathrm{Ru}(\mathrm{bpy})_{3}\right]^{2+}$ for which $E_{o o}$ is $2.1 \mathrm{eV} .^{2}$ Thus, $E^{\mathrm{O}}\left(\mathrm{Ru}^{\mathrm{II} * / \mathrm{I}}\right)=0.82 \mathrm{~V}$ and the free energy change, $\Delta E$, for excited state oxidation of the phenol, $\left(E^{\mathrm{O}}\left(\mathrm{Ru}^{\mathrm{II} / \mathrm{I}}\right)-\right.$ $E^{\mathrm{O}}(\mathrm{PhOH} / \mathrm{PhO})^{\circ}$, is minimally $-0.31 \mathrm{eV}$ using the peak potential of the $\mathrm{PhOH} / \mathrm{PhO}{ }^{*}$ couple. This is not an exact value because the analysis does not consider the effect of the excited state on the measured ground state $\mathrm{PhOH} / \mathrm{PhO}^{\circ}$ potential which should be significant in the strongly coupled system. Nevertheless, with $\Delta G^{\mathrm{o}}=-\mathrm{nFE}$, it is significantly uphill.

Table S1. Summary of photophysical data in acetonitrile.

\begin{tabular}{lcccc} 
& $\tau / \mu \mathrm{s}$ & $\Phi_{\mathrm{em}}$ & $k_{\mathrm{r}}$ & $k_{\mathrm{nr}}$ \\
\hline$\left[\left(\mathrm{bpy}-d_{8}\right)_{2} \mathrm{Ru}(\right.$ phen-Im-PhOMe $\left.)\right]\left(\mathrm{PF}_{6}\right)_{2}$ & 1.50 & 0.098 & $6.5 \times 10^{4} \mathrm{~s}^{-1}$ & $6.0 \times 10^{5} \mathrm{~s}^{-1}$ \\
{$\left[\left(\mathrm{bpy}-d_{8}\right)_{2} \mathrm{Ru}(\right.$ phen-Im-PhOH$\left.)\right]\left(\mathrm{PF}_{6}\right)_{2}$} & 1.48 & 0.099 & $6.2 \times 10^{4} \mathrm{~s}^{-1}$ & $6.1 \times 10^{5} \mathrm{~s}^{-1}$ \\
{$\left[\left(\mathrm{bpy}-d_{8}\right)_{2} \mathrm{Ru}(\right.$ phen-Im-PhOD) $]\left(\mathrm{PF}_{6}\right)_{2}$} & 1.50 & 0.11 & $7.4 \times 10^{4} \mathrm{~s}^{-1}$ & $5.9 \times 10^{5} \mathrm{~s}^{-1}$ \\
\hline
\end{tabular}



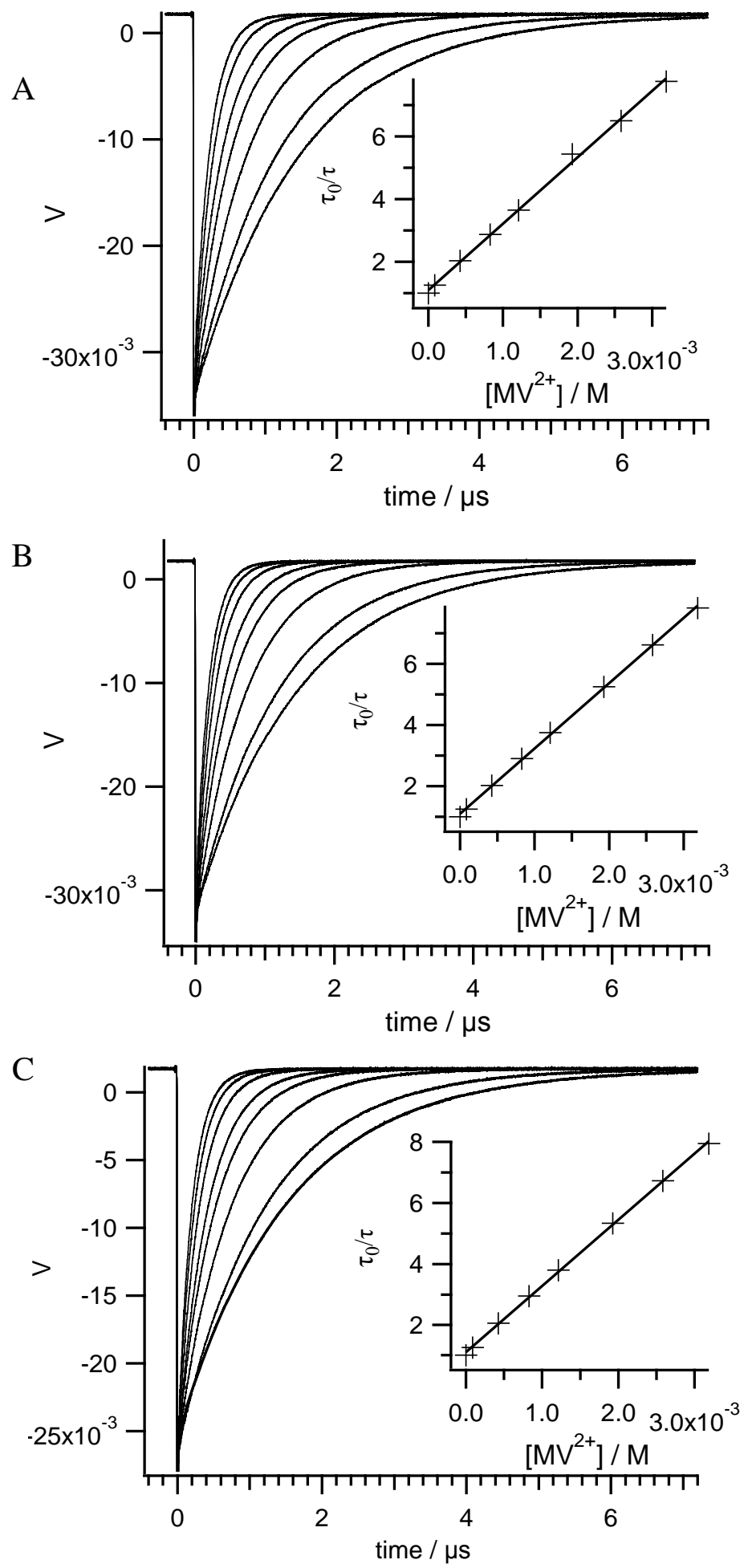

Figure S19. Stern-Volmer quenching of $\left[\left(\mathrm{bpy}-d_{8}\right)_{2} \mathrm{Ru}(\right.$ phen-Im-PhOMe $\left.)\right]\left(\mathrm{PF}_{6}\right)_{2}(\mathrm{~A}),\left[\left(\mathrm{bpy}-d_{8}\right)_{2} \mathrm{Ru}(\mathrm{phen}-\right.$ $\mathrm{Im}-\mathrm{P} h \mathrm{OH})]\left(\mathrm{PF}_{6}\right)_{2}(\mathrm{~B})$ and $\left[\left(\mathrm{bpy}-d_{8}\right)_{2} \mathrm{Ru}(\right.$ phen-Im-PhOD) $]\left(\mathrm{PF}_{6}\right)_{2}(\mathrm{C})$ with methyl viologen in deoxygenated $\mathrm{CH}_{3} \mathrm{CN}$. The quenching constants, $k_{\mathrm{Q}}$, are $1.41 \times 10^{9} \mathrm{M}^{-1} \mathrm{~s}^{-1}, 1.45 \times 10^{9} \mathrm{M}^{-1} \mathrm{~s}^{-1}$, and $1.39 \times 10^{9} \mathrm{M}^{-1} \mathrm{~s}^{-1}$, respectively. 

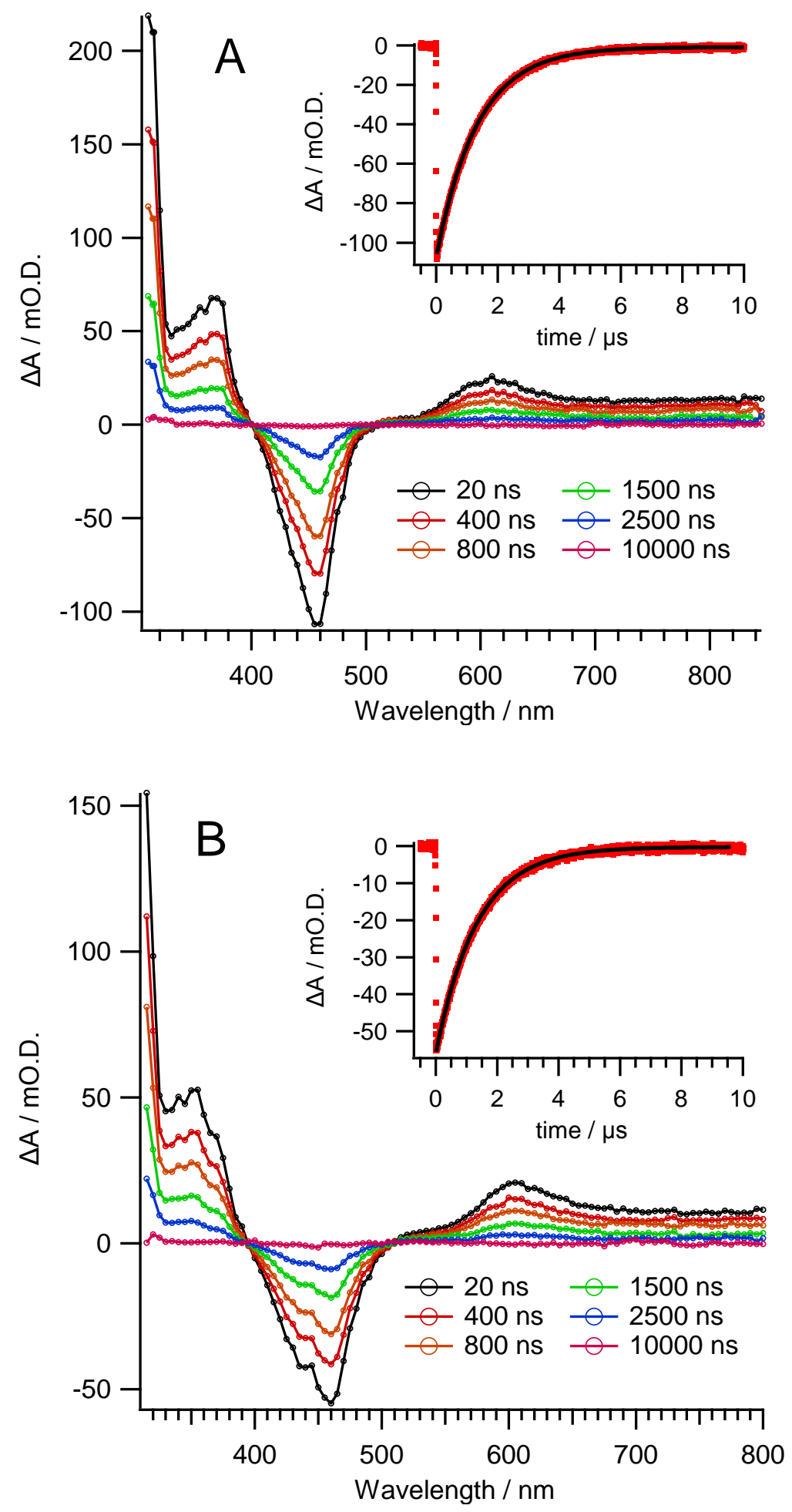

Figure S20. Excited state transient absorption spectra of $\left[\left(\mathrm{bpy}-d_{8}\right)_{2} \mathrm{Ru}(\mathrm{phen}-\mathrm{Im}-\mathrm{PhOMe})\right]\left(\mathrm{PF}_{6}\right)_{2}(\mathrm{~A})$ and $\left[\left(\text { bpy- } d_{8}\right)_{2} \mathrm{Ru}(\right.$ phen-Im-PhOH $\left.)\right]\left(\mathrm{PF}_{6}\right)_{2}(\mathrm{~B})$ in $\mathrm{CH}_{3} \mathrm{CN}$. 

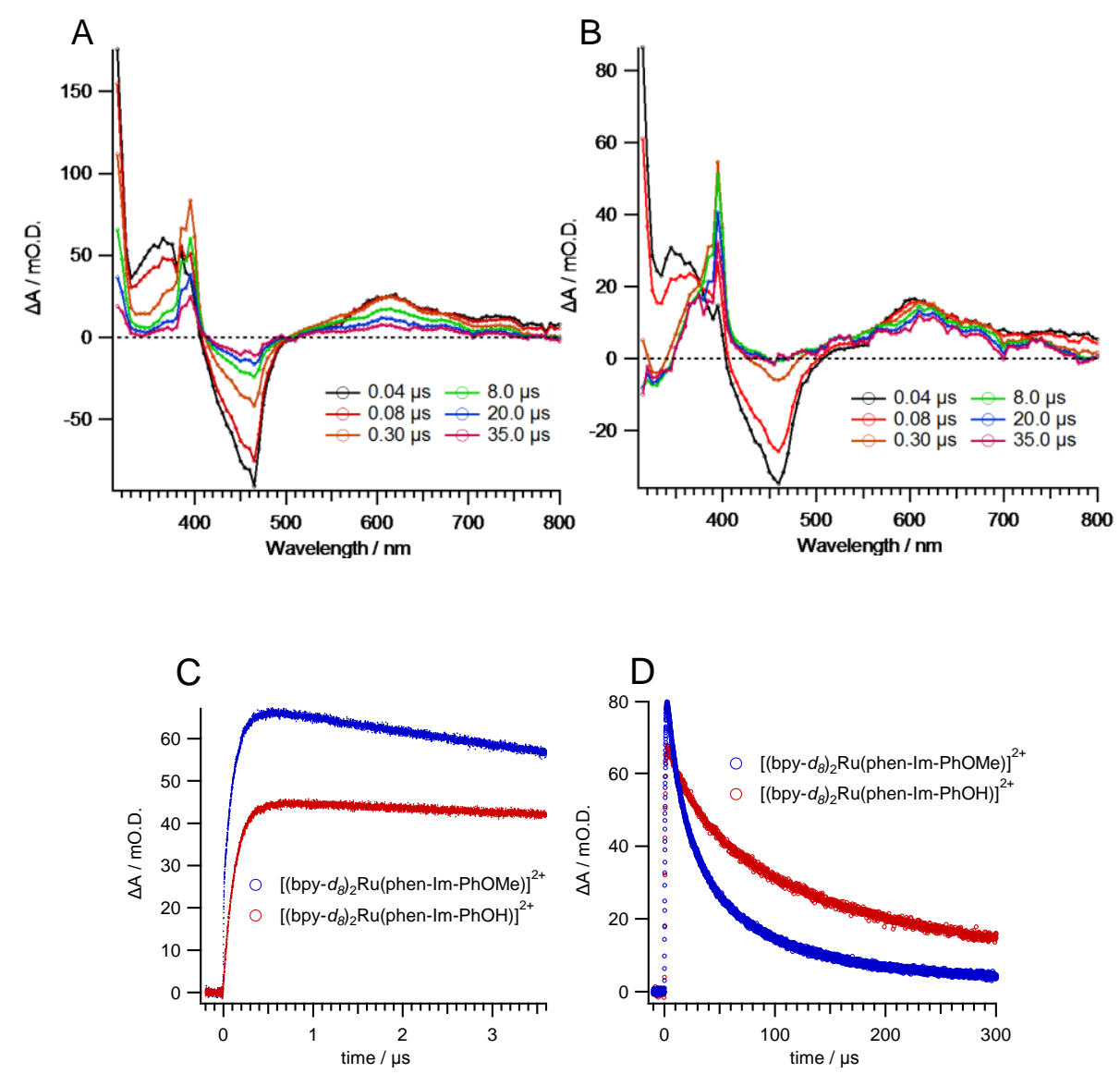

Figure S21. Transient absorption spectra for $\left[\left(\mathrm{bpy}-d_{8}\right)_{2} \mathrm{Ru}(\mathrm{phen}-\mathrm{Im}-\mathrm{PhOMe})\right]\left(\mathrm{PF}_{6}\right)_{2}(\mathrm{~A})$ and $[(\mathrm{bpy}-$ $\left.d_{8}\right)_{2} \mathrm{Ru}($ phen-Im-PhOH $\left.)\right]\left(\mathrm{PF}_{6}\right)_{2}(\mathrm{~B})$ in the presence of $5 \mathrm{mM}$ methyl viologen. The data in $\mathrm{C}$ and $\mathrm{D}$ are the kinetic traces at $390 \mathrm{~nm}$ showing the growth (C) and disappearance (D) of the methyl viologen radical.

In the presence of $5 \mathrm{mM}$ methyl viologen $\left(\mathrm{MV}^{2+}\right)$, the ${ }^{3} \mathrm{MLCT}$ state is quenched oxidatively as shown by loss of the $350 \mathrm{~nm}$ absorbance and growth of methyl viologen radical cation $\left(\mathrm{MV}^{+\bullet}\right)$ signals at 390 and $610 \mathrm{~nm}$. The flash/quench experiment yielded different results for the $\mathrm{PhOMe}$ and $\mathrm{PhOH}$ complexes. Spectra of the PhOMe complex evolve from the ${ }^{3}$ MLCT excited state spectrum at 40 ns to a spectrum at $300 \mathrm{~ns}$ with methyl viologen radical cation $\left(\mathrm{MV}^{+\bullet}\right)$ absorptions at 390 and $610 \mathrm{~nm}$ and the ${ }^{3}$ MLCT bleach of the oxidized $\mathrm{Ru}^{\mathrm{III}}$ at $460 \mathrm{~nm}$. These signals decay over several hundred $\mu$ s according to equal concentration second order kinetics due to the recombination of $\left(\mathrm{MV}^{+\bullet}\right)$ and $\mathrm{Ru}^{\mathrm{III}}$ (Scheme $\left.1 k_{\text {BET_1 }}\right)$. Clear isosbestic points are present during the quenching and recombination reactions.

In contrast, the spectra of Ru-phen-Im-PhOH evolve without isosbestic points from the $40 \mathrm{~ns}$ excited state to spectra after $300 \mathrm{~ns}$ which contain $\mathrm{MV}^{+\bullet}$ absorptions but not the $460 \mathrm{~nm}$ bleach associated with the $[\mathrm{Ru}]^{3+}$. The $\mathrm{MV}^{+\bullet}$ signals again decay according to second order kinetics but more slowly than the recombination with the $\mathrm{Ru}^{\mathrm{III}}$ of the PhOMe complex. The disappearance of the $\mathrm{Ru}^{\mathrm{III}}$ bleach following oxidation by methyl viologen is consistent with intramolecular oxidation of the phenol by $\mathrm{Ru}^{\mathrm{III}}$. The kinetics of the $\mathrm{Ru}^{\mathrm{III}}$ bleach disappearance (at $5 \mathrm{mM} \mathrm{MV}^{2+}$ ) match the growth of the $\mathrm{MV}^{+\bullet}$ absorptions indicating that the quenching reaction is rate limiting. The slower kinetics for $\mathrm{MV}^{+\bullet}$ disappearance are consistent with a lower driving force for recombination with the phenoxyl radical (Scheme $1 k_{\mathrm{BET}_{-} 2}$ ). 


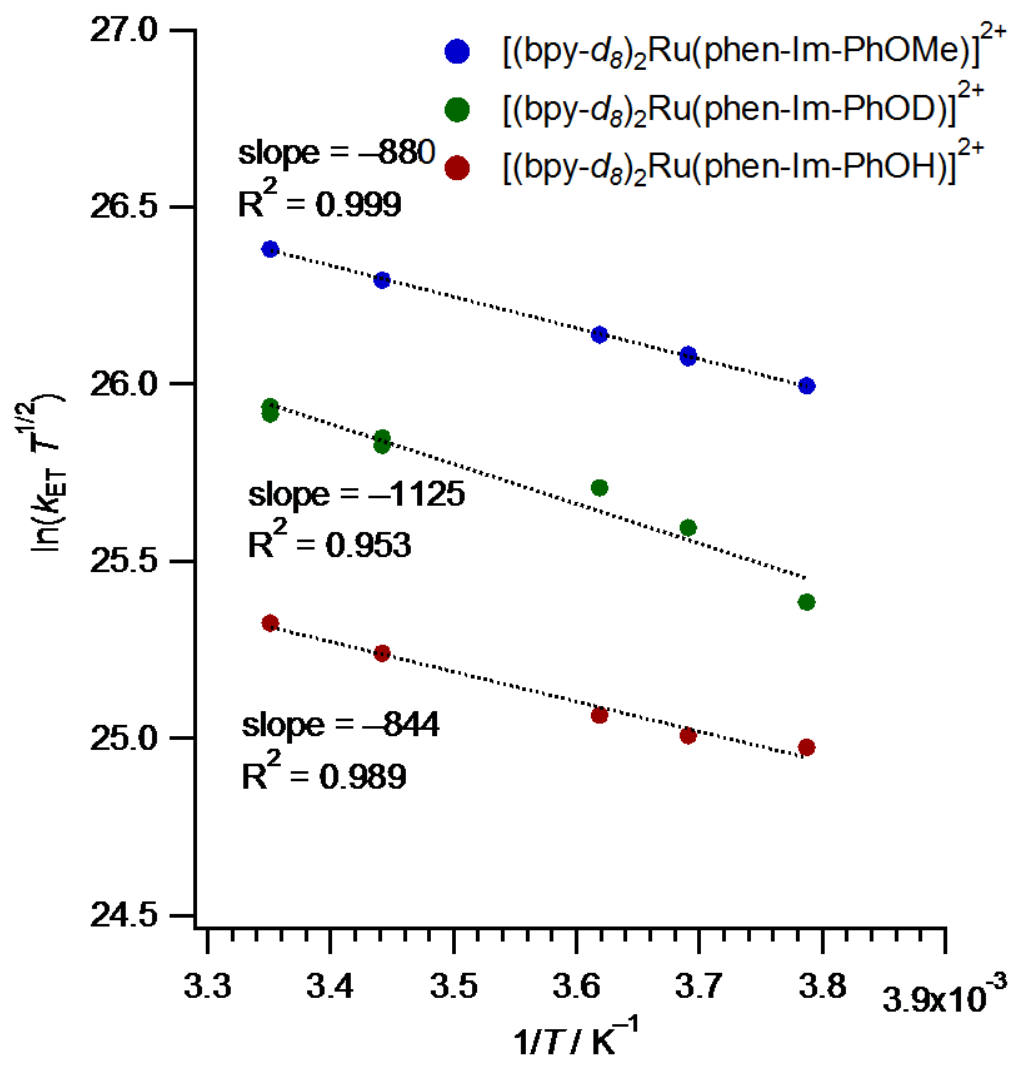

Figure S22. Temperature dependence of the recombination reaction between methyl viologen radicals and $\left[\left(\mathrm{bpy}-d_{8}\right)_{2} \mathrm{Ru}{ }^{\mathrm{III}}(\text { phen-Im-PhOMe })\right]^{3+}$ or $\left[\left(\mathrm{bpy}-d_{8}\right)_{2} \mathrm{Ru}{ }^{\mathrm{II}}(\text { phen-Im(H/D)-PhO} \cdot)\right]^{3+}$ in $\mathrm{CH}_{3} \mathrm{CN}$. 
Table S2. Kinetic data for intramolecular phenol oxidation obtained from decay of the $460 \mathrm{~nm}$ bleach in transient absorption data.

\begin{tabular}{|c|c|c|}
\hline Temperature, ${ }^{\circ} \mathrm{C}$ & $k_{\mathrm{ET}}$ Ru-phen-Im-PhOH, ${ }^{-1}$ & $k_{\mathrm{ET}}$ Ru-phen-Im-PhOD, $\mathrm{s}^{-1}$ \\
\hline-10 & $6.398 \mathrm{E}+06$ & $6.566 \mathrm{E}+06$ \\
\hline-5.2 & $8.703 \mathrm{E}+06$ & $9.024 \mathrm{E}+06$ \\
\hline 0.8 & $1.164 \mathrm{E}+07$ & $1.202 \mathrm{E}+07$ \\
\hline 4.4 & $1.403 \mathrm{E}+07$ & $1.404 \mathrm{E}+07$ \\
\hline-12.5 & $5.647 \mathrm{E}+06$ & $5.832 \mathrm{E}+06$ \\
\hline-9.6 & $6.751 \mathrm{E}+06$ & $6.991 \mathrm{E}+06$ \\
\hline-2.7 & $9.748 \mathrm{E}+06$ & $1.011 \mathrm{E}+07$ \\
\hline-0.5 & $1.116 \mathrm{E}+07$ & $1.131 \mathrm{E}+07$ \\
\hline 2.2 & $1.301 \mathrm{E}+07$ & $1.301 \mathrm{E}+07$ \\
\hline 5.9 & $1.583 \mathrm{E}+07$ & $1.562 \mathrm{E}+07$ \\
\hline 10.5 & $1.938 \mathrm{E}+07$ & $1.971 \mathrm{E}+07$ \\
\hline 15.2 & $2.534 \mathrm{E}+07$ & $2.361 \mathrm{E}+07$ \\
\hline 2 & $1.223 \mathrm{E}+07$ & $1.206 \mathrm{E}+07$ \\
\hline 5.6 & $1.532 \mathrm{E}+07$ & $1.473 \mathrm{E}+07$ \\
\hline 9.7 & $1.801 \mathrm{E}+07$ & $1.719 \mathrm{E}+07$ \\
\hline 15.5 & $2.214 \mathrm{E}+07$ & $2.270 \mathrm{E}+07$ \\
\hline 19 & $2.625 \mathrm{E}+07$ & $2.762 \mathrm{E}+07$ \\
\hline
\end{tabular}

Table S3. Kinetic data for phenoxyl radical reduction by $\mathrm{MV}^{\bullet+}$. The data are $k_{\mathrm{obs}}$ not converted to $k_{\mathrm{BET}_{-} 1}($ see text).

\begin{tabular}{|c|c|c|c|}
\hline $\begin{array}{c}\text { Temperature } \\
{ }^{\circ} \mathrm{C}\end{array}$ & $\begin{array}{c}k_{\mathrm{ET}} \text { Ru-phen-Im-PhOH } \\
\mathrm{M}^{-1} \mathrm{~s}^{-1}\end{array}$ & $\begin{array}{c}k_{\mathrm{ET}} \text { Ru-phen-Im-PhOD } \\
\mathrm{M}^{-1} \mathrm{~s}^{-1}\end{array}$ & $\begin{array}{c}k_{\mathrm{ET}} \text { Ru-phen-Im-PhOMe } \\
\mathrm{M}^{-1} \mathrm{~s}^{-1}\end{array}$ \\
\hline-9.1 & $3.18 \mathrm{E}+09$ & $4.22 \mathrm{E}+09$ & $1.20 \mathrm{E}+10$ \\
\hline-2.2 & $3.29 \mathrm{E}+09$ & $4.91 \mathrm{E}+09$ & $1.29 \mathrm{E}+10$ \\
& & & $1.28 \mathrm{E}+10$ \\
\hline 3.2 & $3.45 \mathrm{E}+09$ & $5.33 \mathrm{E}+09$ & $1.35 \mathrm{E}+10$ \\
\hline 17.4 & $3.98 \mathrm{E}+09$ & $5.94 \mathrm{E}+09$ & $1.54 \mathrm{E}+10$ \\
& & $6.02 \mathrm{E}+09$ & $1.66 \mathrm{E}+10$ \\
\hline 25.3 & $4.28 \mathrm{E}+09$ & $6.48 \mathrm{E}+09$ & \\
\hline
\end{tabular}




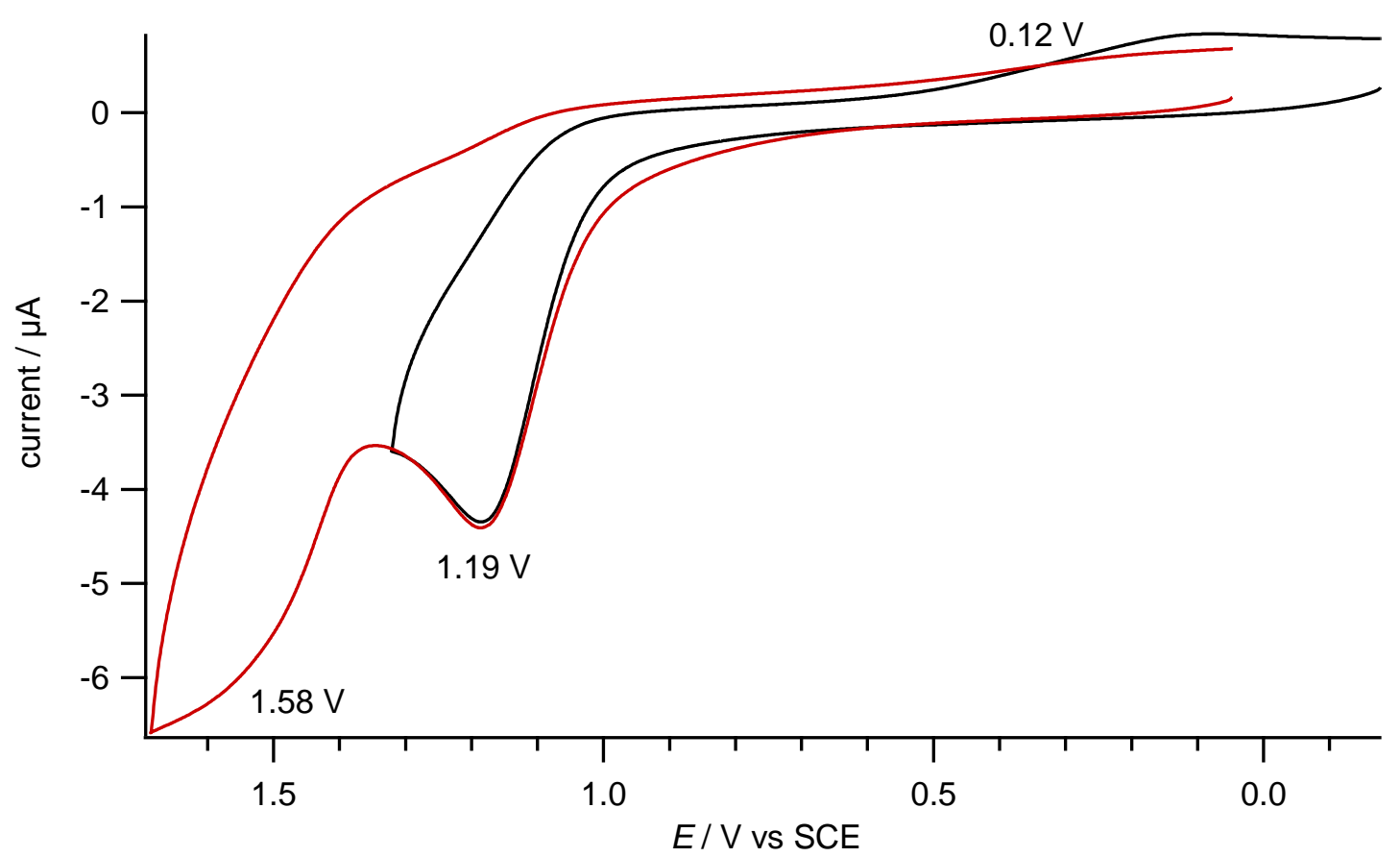

Figure S23. Cyclic voltammograms of phen-Im- $\mathrm{PhOH}$ in $\mathrm{CH}_{2} \mathrm{Cl}_{2}$.

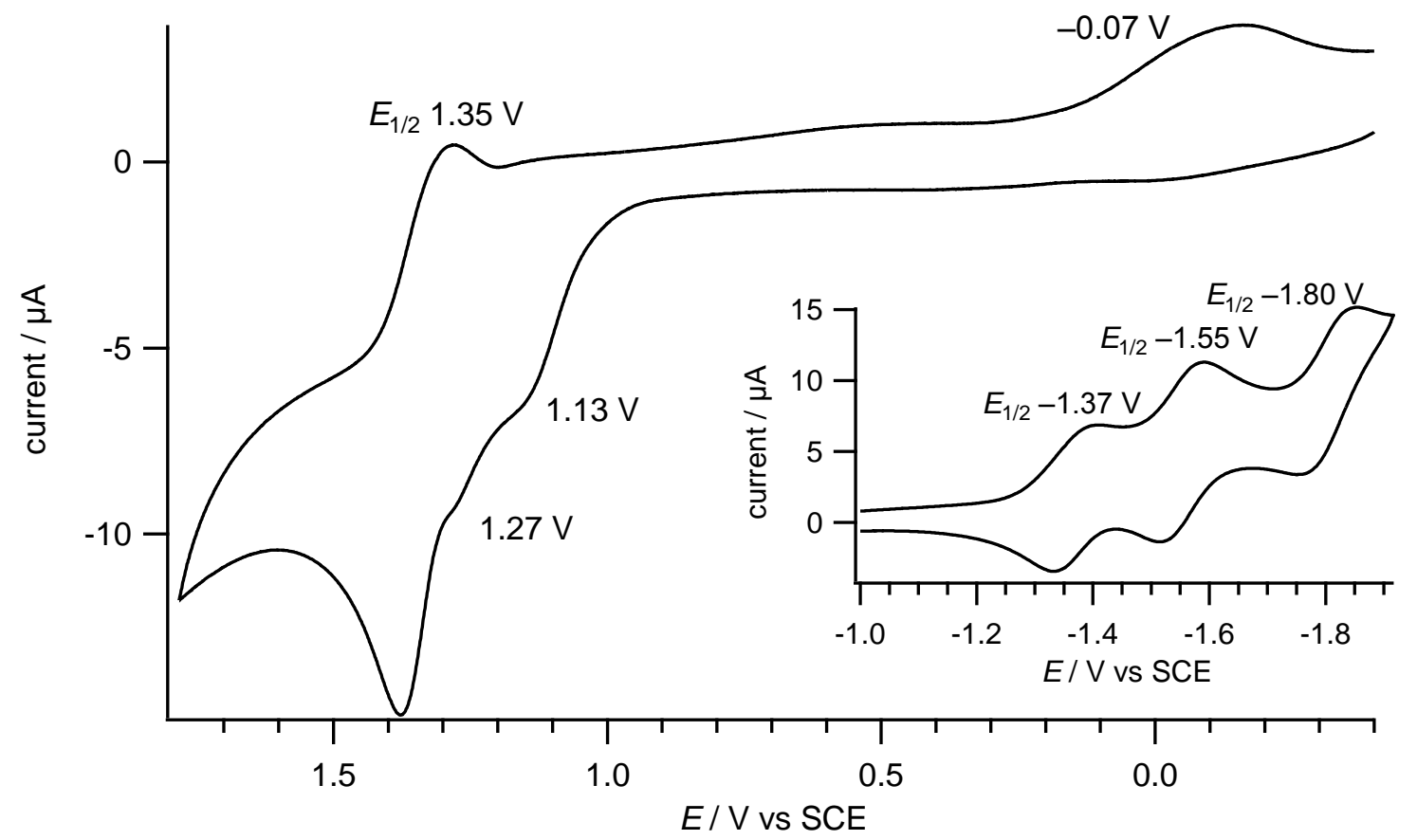

Figure S24. Cyclic voltammograms of $\left[(\mathrm{bpy})_{2} \mathrm{Ru}^{\mathrm{II}}(\text { phen-Im-PhOH})\right]^{2+}$ in $\mathrm{CH}_{3} \mathrm{CN}$. 


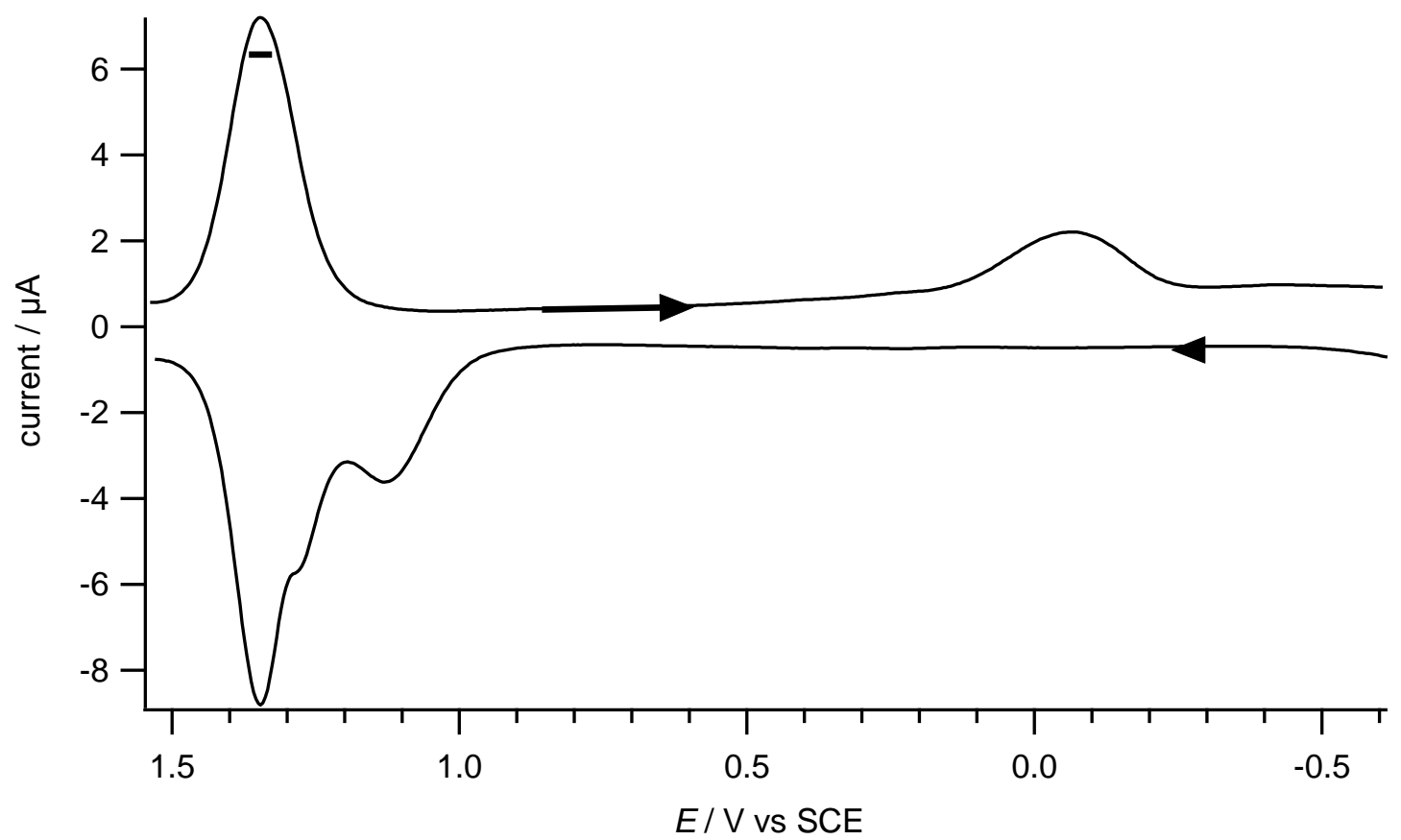

Figure S25. Square wave voltammograms of $\left[(\mathrm{bpy}){ }_{2} \mathrm{Ru}{ }^{\mathrm{II}}(\text { phen-Im-PhOH})\right]^{2+}$ in $\mathrm{CH}_{3} \mathrm{CN}$.

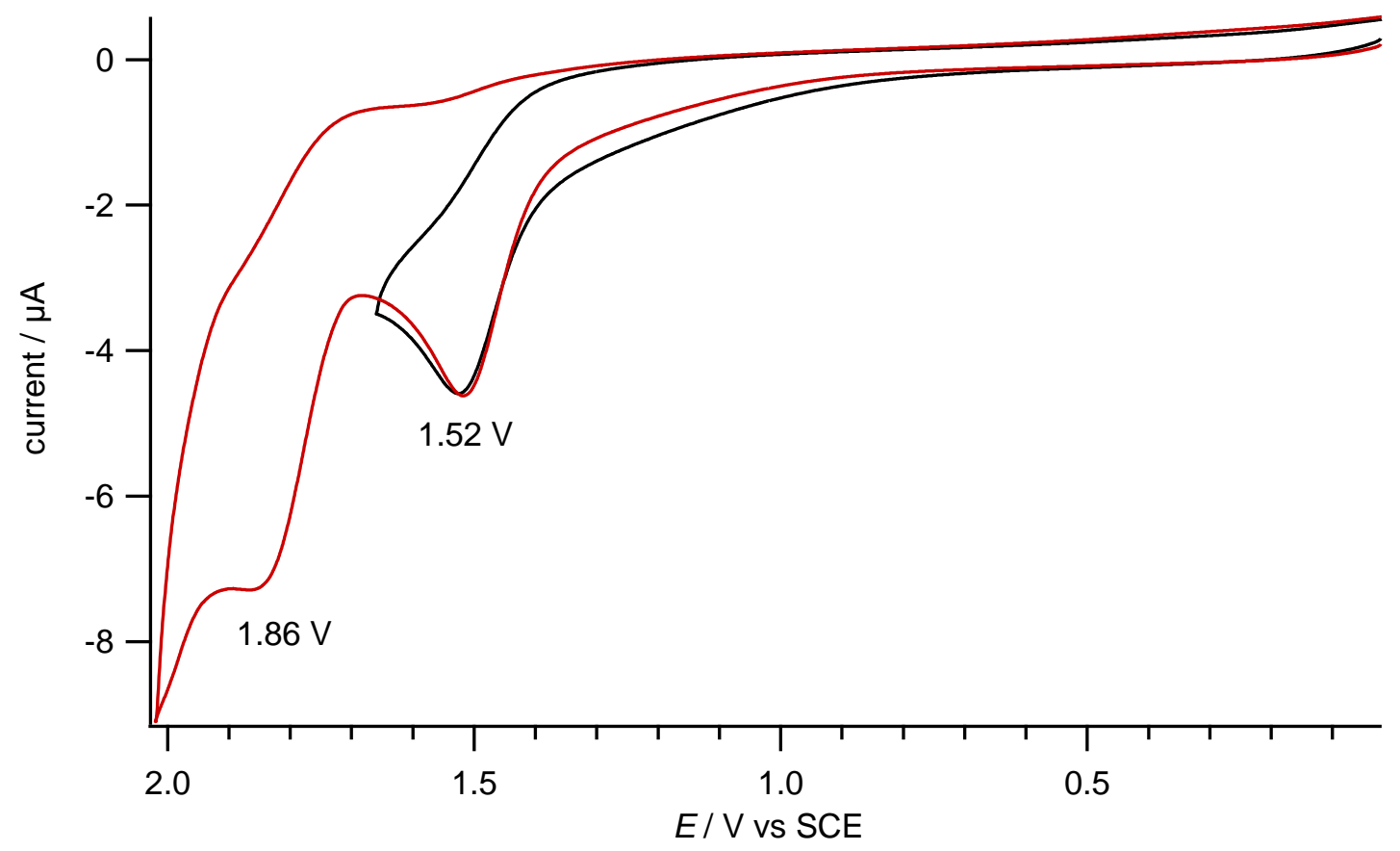

Figure S26. Cyclic voltammograms of phen-Im-PhOMe in $\mathrm{CH}_{2} \mathrm{Cl}_{2}$. 


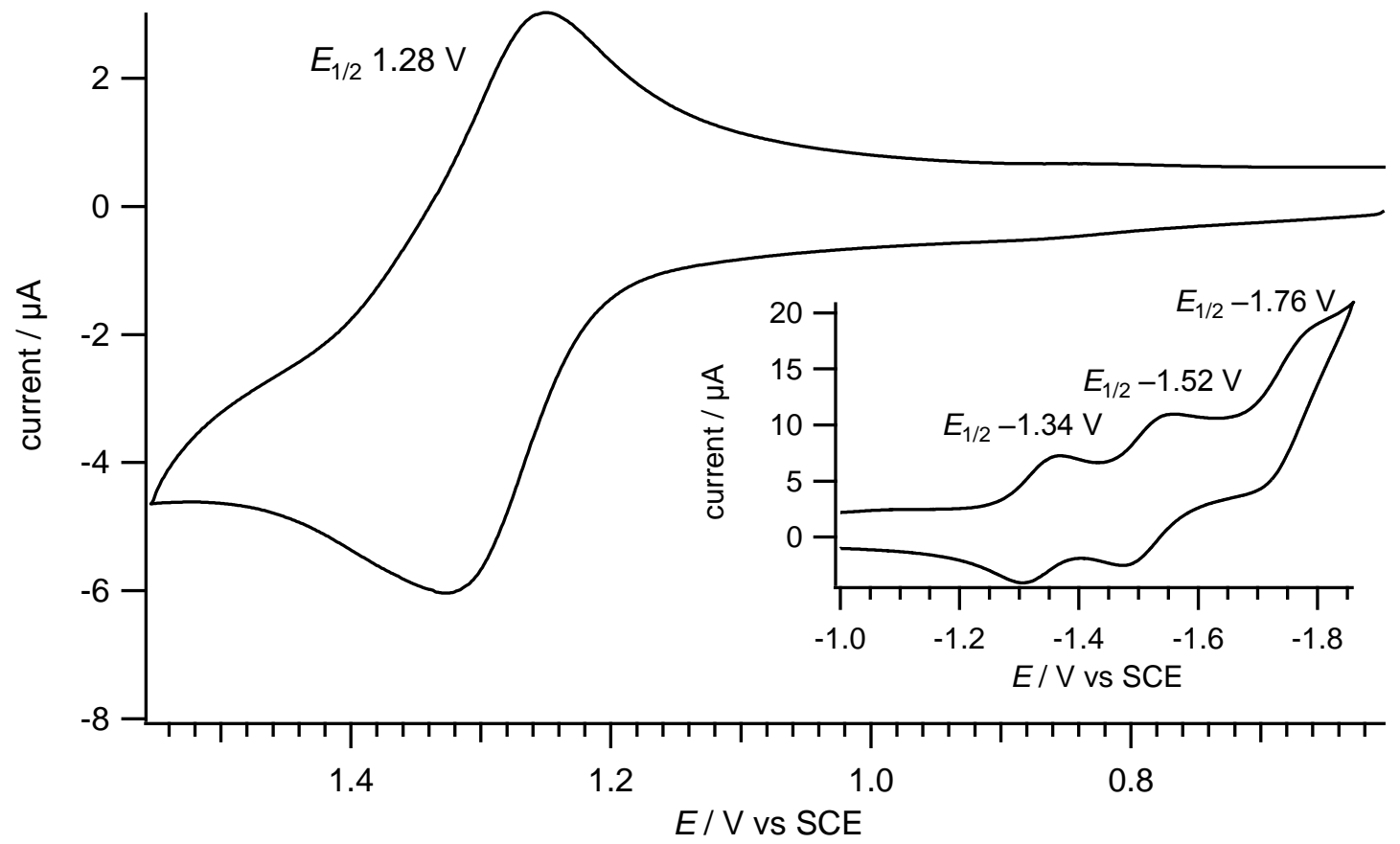

Figure S27. Cyclic voltammograms of $\left[(\mathrm{bpy})_{2} \mathrm{Ru}^{\mathrm{II}}(\text { phen-Im-PhOMe })\right]^{2+}$ in $\mathrm{CH}_{3} \mathrm{CN}$. 
Table S4. Summary of redox potentials in volts vs SCE. The ligands were analyzed in $\mathrm{CH}_{2} \mathrm{Cl}_{2}$ and complexes were measured in $\mathrm{CH}_{3} \mathrm{CN}$. Phenol redox couples are shown in bold font.

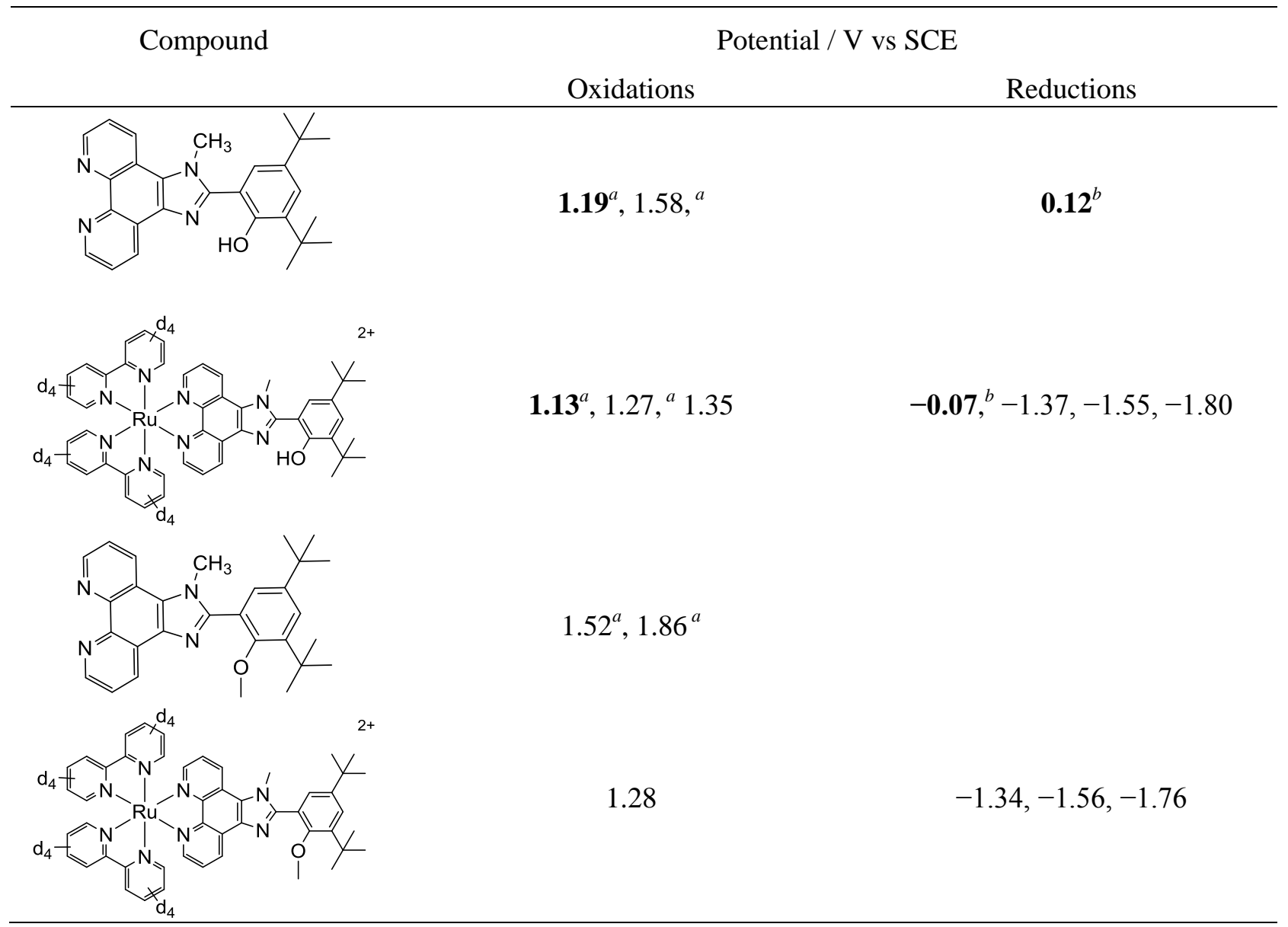

${ }^{a}$ Anodic peak potential for an irreversible wave.

${ }^{b}$ Cathodic peak potential for an irreversible wave. 
Table S5. Predicted stretching O-H and O-D frequencies from DFT calculations for the $\mathrm{Ru}^{\mathrm{III}}$ complex obtained immediately after oxidative quenching with methyl viologen and stretching $\mathrm{N}-\mathrm{H}$ and $\mathrm{N}-\mathrm{D}$ frequencies for the phenoxyl radicals generated after intramolecular proton coupled electron transfer. ${ }^{a}$

\begin{tabular}{|cc|}
\hline$\left[(\text { bpy })_{2} \mathrm{Ru}^{\mathrm{III}}(\text { phen-Im-PhOH})\right]^{3+}$ & {$\left[(\mathrm{bpy}){ }_{2} \mathrm{Ru}^{\mathrm{II}}(\mathrm{phen}-\mathrm{Im}(\mathrm{H})-\mathrm{PhO} \cdot)\right]^{3+}$} \\
$\mathrm{N}---\mathrm{H}-\mathrm{OPh} 3392 \mathrm{~cm}^{-1}$ & $\mathrm{~N}-\mathrm{H}---\mathrm{OPh} 3454 \mathrm{~cm}^{-1}$ \\
{$\left[(\mathrm{bpy}){ }_{2} \mathrm{Ru}^{\mathrm{III}}(\text { phen-Im-PhOD})\right]^{3+}$} & {$\left[(\mathrm{bpy}){ }_{2} \mathrm{Ru} \text { II }^{\mathrm{II}}(\mathrm{phen}-\mathrm{Im}(\mathrm{D})-\mathrm{PhO} \cdot)\right]^{3+}$} \\
$\mathrm{N}---\mathrm{D}-\mathrm{OPh} 2472 \mathrm{~cm}^{-1}$ & $\mathrm{~N}-\mathrm{D}---\mathrm{OPh} 2542 \mathrm{~cm}^{-1}$ \\
\hline
\end{tabular}

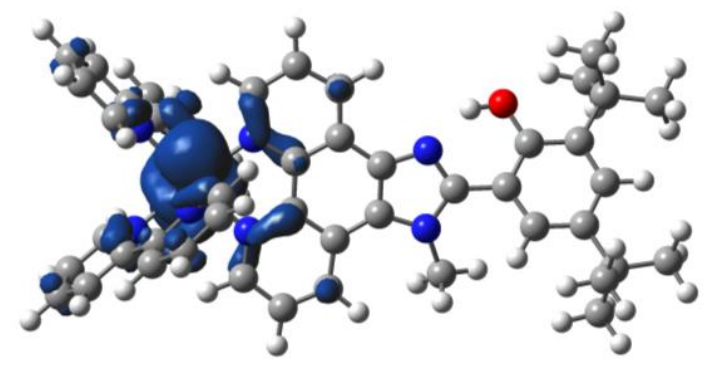

A

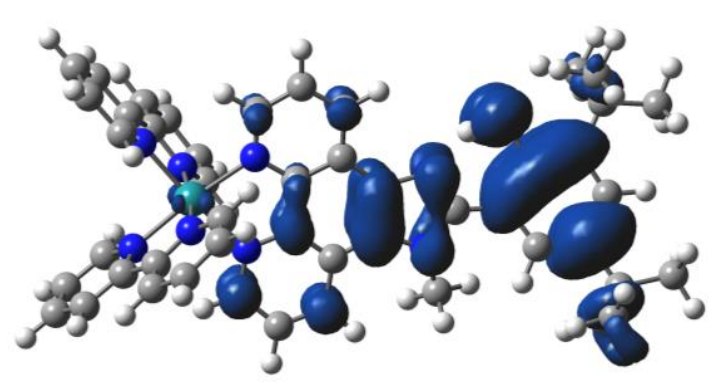

B

Figure S28. DFT calculated spin densities for the $\mathrm{Ru}^{\text {III }}$ complex obtained immediately after oxidative quenching with methyl viologen (A) and the phenoxyl radicals observed after intramolecular proton coupled electron transfer (B).

Table S6. Calculated XYZ coordinates of $\left[(\mathrm{bpy}){ }_{2} \mathrm{Ru} \text { (II }(\mathrm{phen}-\mathrm{Im}-\mathrm{PhOH})\right]^{2+}$

\begin{tabular}{|c|c|c|c|}
\hline Atom & $\mathrm{X}$ & $\mathrm{Y}$ & $\mathrm{Z}$ \\
\hline $\mathrm{H}$ & -2.320996 & 2.451933 & -4.476015 \\
\hline $\mathrm{C}$ & -2.924885 & 2.496019 & -3.579573 \\
\hline $\mathrm{H}$ & -3.922793 & 4.344824 & -4.065635 \\
\hline $\mathrm{C}$ & -3.812787 & 3.541217 & -3.348674 \\
\hline $\mathrm{N}$ & -3.53822 & 1.482289 & -1.49571 \\
\hline $\mathrm{C}$ & -4.561074 & 3.540605 & -2.179348 \\
\hline $\mathrm{C}$ & -2.817525 & 1.490039 & -2.630934 \\
\hline $\mathrm{C}$ & -4.410659 & 2.50007 & -1.262123 \\
\hline $\mathrm{H}$ & -2.142343 & 0.65854 & -2.774791 \\
\hline $\mathrm{C}$ & -6.117192 & 3.351876 & 0.399672 \\
\hline $\mathrm{C}$ & -6.774469 & 3.195437 & 1.612595 \\
\hline $\mathrm{C}$ & -5.163293 & 2.413476 & 0.003719 \\
\hline $\mathrm{C}$ & -6.463066 & 2.099078 & 2.409741 \\
\hline $\mathrm{H}$ & -7.516202 & 3.918257 & 1.927677 \\
\hline
\end{tabular}




\begin{tabular}{|c|c|c|c|}
\hline $\mathrm{H}$ & -6.947284 & 1.93459 & 3.36301 \\
\hline $\mathrm{C}$ & -5.50768 & 1.198832 & 1.962691 \\
\hline $\mathrm{H}$ & -5.236715 & 0.335831 & 2.554255 \\
\hline $\mathrm{N}$ & -4.866967 & 1.34278 & 0.789327 \\
\hline $\mathrm{Ru}$ & -3.420489 & 0.011798 & 0.017313 \\
\hline $\mathrm{H}$ & -7.197178 & -1.660352 & -3.186683 \\
\hline $\mathrm{C}$ & -6.688044 & -1.859824 & -2.253272 \\
\hline $\mathrm{H}$ & -7.839319 & -3.607396 & -1.73258 \\
\hline $\mathrm{C}$ & -7.03989 & -2.936175 & -1.445776 \\
\hline $\mathrm{N}$ & -4.982435 & -1.217252 & -0.696359 \\
\hline $\mathrm{C}$ & -6.347701 & -3.140062 & -0.259691 \\
\hline $\mathrm{C}$ & -5.657655 & -1.027086 & -1.843531 \\
\hline $\mathrm{C}$ & -5.319484 & -2.267818 & 0.099944 \\
\hline $\mathrm{H}$ & -5.35273 & -0.182035 & -2.444359 \\
\hline $\mathrm{C}$ & -4.713282 & -3.435563 & 2.26058 \\
\hline $\mathrm{C}$ & -3.9201 & -3.490622 & 3.398582 \\
\hline $\mathrm{C}$ & -4.526547 & -2.407306 & 1.336033 \\
\hline $\mathrm{C}$ & -2.95105 & -2.511768 & 3.591078 \\
\hline $\mathrm{H}$ & -4.057807 & -4.284992 & 4.121018 \\
\hline $\mathrm{H}$ & -2.308947 & -2.51214 & 4.461667 \\
\hline $\mathrm{C}$ & -2.812004 & -1.514233 & 2.637799 \\
\hline $\mathrm{H}$ & -2.073579 & -0.733448 & 2.752881 \\
\hline $\mathrm{N}$ & -3.577025 & -1.452924 & 1.533524 \\
\hline $\mathrm{H}$ & -6.347642 & 4.197966 & -0.231351 \\
\hline $\mathrm{H}$ & -5.25387 & 4.346251 & -1.984497 \\
\hline $\mathrm{H}$ & -5.469412 & -4.1892 & 2.095382 \\
\hline $\mathrm{H}$ & -6.60854 & -3.971732 & 0.378701 \\
\hline $\mathrm{H}$ & -0.960507 & -3.746421 & -2.69732 \\
\hline $\mathrm{C}$ & -0.816619 & -2.868745 & -2.081577 \\
\hline $\mathrm{H}$ & 1.307564 & -2.977394 & -2.118844 \\
\hline $\mathrm{C}$ & 0.450189 & -2.429254 & -1.759943 \\
\hline $\mathrm{N}$ & -1.823557 & -1.101515 & -0.824259 \\
\hline $\mathrm{C}$ & 0.606774 & -1.281202 & -0.955718 \\
\hline $\mathrm{C}$ & -1.932741 & -2.177524 & -1.604804 \\
\hline $\mathrm{C}$ & -0.580124 & -0.651415 & -0.485164 \\
\hline $\mathrm{C}$ & 1.841212 & -0.688923 & -0.529746 \\
\hline $\mathrm{H}$ & -2.934432 & -2.49915 & -1.85333 \\
\hline $\mathrm{C}$ & 1.884253 & 0.399945 & 0.336386 \\
\hline $\mathrm{C}$ & 0.696854 & 1.026294 & 0.827523 \\
\hline $\mathrm{C}$ & 0.678257 & 2.127775 & 1.701289 \\
\hline $\mathrm{C}$ & -0.534012 & 0.490118 & 0.393456 \\
\hline $\mathrm{C}$ & -0.538089 & 2.642384 & 2.09708 \\
\hline
\end{tabular}




\begin{tabular}{|c|c|c|c|}
\hline $\mathrm{H}$ & 1.610822 & 2.552176 & 2.050135 \\
\hline $\mathrm{H}$ & -0.599324 & 3.489566 & 2.767161 \\
\hline $\mathrm{C}$ & -1.721313 & 2.058183 & 1.627956 \\
\hline $\mathrm{H}$ & -2.68542 & 2.441776 & 1.931837 \\
\hline $\mathrm{N}$ & -1.729033 & 1.00989 & 0.802938 \\
\hline $\mathrm{N}$ & 3.17734 & 0.734712 & 0.612829 \\
\hline $\mathrm{N}$ & 3.165003 & -0.999791 & -0.801942 \\
\hline $\mathrm{C}$ & 3.936479 & -0.123384 & -0.064683 \\
\hline $\mathrm{C}$ & 5.400487 & -0.085721 & -0.009292 \\
\hline $\mathrm{C}$ & 8.162769 & 0.040595 & 0.059641 \\
\hline $\mathrm{C}$ & 6.045061 & 1.15736 & 0.226054 \\
\hline $\mathrm{C}$ & 6.172248 & -1.250301 & -0.128658 \\
\hline $\mathrm{C}$ & 7.562595 & -1.216241 & -0.093492 \\
\hline $\mathrm{C}$ & 7.454326 & 1.234976 & 0.226696 \\
\hline $\mathrm{H}$ & 5.663356 & -2.200519 & -0.217409 \\
\hline $\mathrm{H}$ & 9.239637 & 0.091366 & 0.071576 \\
\hline $\mathrm{O}$ & 5.322436 & 2.28381 & 0.444607 \\
\hline $\mathrm{H}$ & 4.389084 & 2.019702 & 0.613999 \\
\hline $\mathrm{C}$ & 3.630901 & -1.889251 & -1.869379 \\
\hline $\mathrm{H}$ & 4.643173 & -1.60961 & -2.144377 \\
\hline $\mathrm{H}$ & 3.618137 & -2.932764 & -1.551091 \\
\hline $\mathrm{H}$ & 2.994585 & -1.759609 & -2.743145 \\
\hline $\mathrm{C}$ & 8.186644 & 2.580412 & 0.425991 \\
\hline $\mathrm{C}$ & 7.850842 & 3.166374 & 1.819193 \\
\hline $\mathrm{H}$ & 8.169692 & 2.480927 & 2.610096 \\
\hline $\mathrm{H}$ & 6.783641 & 3.349957 & 1.931512 \\
\hline $\mathrm{H}$ & 8.381692 & 4.112961 & 1.961102 \\
\hline $\mathrm{C}$ & 7.775221 & 3.578813 & -0.683295 \\
\hline $\mathrm{H}$ & 8.039723 & 3.186487 & -1.669854 \\
\hline $\mathrm{H}$ & 8.306703 & 4.525486 & -0.545328 \\
\hline $\mathrm{H}$ & 6.705457 & 3.780618 & -0.66816 \\
\hline $\mathrm{C}$ & 9.71851 & 2.421779 & 0.351846 \\
\hline $\mathrm{H}$ & 10.045661 & 2.029298 & -0.614939 \\
\hline $\mathrm{H}$ & 10.10506 & 1.76839 & 1.138586 \\
\hline $\mathrm{H}$ & 10.182766 & 3.402533 & 0.483094 \\
\hline $\mathrm{C}$ & 8.371977 & -2.52318 & -0.200899 \\
\hline $\mathrm{C}$ & 8.01867 & -3.251331 & -1.518574 \\
\hline $\mathrm{H}$ & 8.247155 & -2.623426 & -2.384305 \\
\hline $\mathrm{H}$ & 6.960457 & -3.519261 & -1.565745 \\
\hline $\mathrm{H}$ & 8.59833 & -4.175098 & -1.605991 \\
\hline $\mathrm{C}$ & 8.025205 & -3.439044 & 0.996293 \\
\hline $\mathrm{H}$ & 8.283117 & -2.955898 & 1.942954 \\
\hline
\end{tabular}




\begin{tabular}{|c|c|c|c|}
\hline $\mathrm{H}$ & 8.584124 & -4.377518 & 0.930167 \\
\hline $\mathrm{H}$ & 6.960935 & -3.685393 & 1.022825 \\
\hline $\mathrm{C}$ & 9.891248 & -2.272746 & -0.187209 \\
\hline $\mathrm{H}$ & 10.217854 & -1.795374 & 0.740511 \\
\hline $\mathrm{H}$ & 10.209386 & -1.647459 & -1.026 \\
\hline $\mathrm{H}$ & 10.416618 & -3.227806 & -0.268389 \\
\hline
\end{tabular}

Table S7. Calculated XYZ coordinates of $\left[(\mathrm{bpy})_{2} \mathrm{Ru}{ }^{\mathrm{III}}(\mathrm{phen}-\mathrm{Im}-\mathrm{PhOH})\right]^{3+}$

\begin{tabular}{|c|c|c|c|}
\hline Atom & $\mathrm{X}$ & $\mathrm{Y}$ & $\mathrm{Z}$ \\
\hline $\mathrm{H}$ & -2.03063 & 2.729011 & -4.20454 \\
\hline $\mathrm{C}$ & -2.69676 & 2.716026 & -3.35299 \\
\hline $\mathrm{H}$ & -3.68768 & 4.57341 & -3.80822 \\
\hline $\mathrm{C}$ & -3.61667 & 3.733942 & -3.1288 \\
\hline $\mathrm{N}$ & -3.44864 & 1.587369 & -1.38578 \\
\hline $\mathrm{C}$ & -4.44704 & 3.666645 & -2.01724 \\
\hline $\mathrm{C}$ & -2.63948 & 1.659756 & -2.4579 \\
\hline $\mathrm{C}$ & -4.34736 & 2.58219 & -1.14973 \\
\hline $\mathrm{H}$ & -1.94439 & 0.845119 & -2.60051 \\
\hline $\mathrm{C}$ & -6.1407 & 3.328511 & 0.468918 \\
\hline $\mathrm{C}$ & -6.86442 & 3.083157 & 1.629055 \\
\hline $\mathrm{C}$ & -5.16675 & 2.4203 & 0.061437 \\
\hline $\mathrm{C}$ & -6.60223 & 1.931835 & 2.362718 \\
\hline $\mathrm{H}$ & -7.6231 & 3.783721 & 1.95308 \\
\hline $\mathrm{H}$ & -7.14064 & 1.701695 & 3.271698 \\
\hline $\mathrm{C}$ & -5.62379 & 1.060748 & 1.910496 \\
\hline $\mathrm{H}$ & -5.38761 & 0.158489 & 2.455811 \\
\hline $\mathrm{N}$ & -4.91786 & 1.298436 & 0.791174 \\
\hline $\mathrm{Ru}$ & -3.42068 & 0.012668 & 0.024855 \\
\hline $\mathrm{H}$ & -7.29888 & -1.35523 & -3.18826 \\
\hline $\mathrm{C}$ & -6.76955 & -1.6322 & -2.28705 \\
\hline $\mathrm{H}$ & -7.92697 & -3.40175 & -1.87645 \\
\hline $\mathrm{C}$ & -7.11237 & -2.76465 & -1.55729 \\
\hline $\mathrm{N}$ & -5.02404 & -1.14202 & -0.73067 \\
\hline $\mathrm{C}$ & -6.39797 & -3.07297 & -0.40664 \\
\hline $\mathrm{C}$ & -5.7217 & -0.84228 & -1.84072 \\
\hline $\mathrm{C}$ & -5.35123 & -2.24719 & -0.00551 \\
\hline $\mathrm{H}$ & -5.4231 & 0.043122 & -2.38257 \\
\hline $\mathrm{C}$ & -4.7095 & -3.56324 & 2.057513 \\
\hline $\mathrm{C}$ & -3.88171 & -3.6972 & 3.165274 \\
\hline $\mathrm{C}$ & -4.53684 & -2.48136 & 1.197953 \\
\hline $\mathrm{C}$ & -2.89165 & -2.74814 & 3.39399 \\
\hline
\end{tabular}




\begin{tabular}{|c|c|c|c|}
\hline $\mathrm{H}$ & -4.00928 & -4.53465 & 3.838897 \\
\hline $\mathrm{H}$ & -2.22567 & -2.81378 & 4.243214 \\
\hline $\mathrm{C}$ & -2.76099 & -1.69342 & 2.504849 \\
\hline $\mathrm{H}$ & -2.00695 & -0.93317 & 2.647414 \\
\hline $\mathrm{N}$ & -3.56556 & -1.5584 & 1.435719 \\
\hline $\mathrm{H}$ & -6.33562 & 4.219155 & -0.11015 \\
\hline $\mathrm{H}$ & -5.16279 & 4.45324 & -1.82973 \\
\hline $\mathrm{H}$ & -5.48007 & -4.29538 & 1.86743 \\
\hline $\mathrm{H}$ & -6.65695 & -3.9489 & 0.169909 \\
\hline $\mathrm{H}$ & -0.97776 & -3.74006 & -2.69816 \\
\hline $\mathrm{C}$ & -0.83195 & -2.86762 & -2.07641 \\
\hline $\mathrm{H}$ & 1.289492 & -2.97525 & -2.12299 \\
\hline $\mathrm{C}$ & 0.434785 & -2.42836 & -1.75572 \\
\hline $\mathrm{N}$ & -1.82409 & -1.11208 & -0.80692 \\
\hline $\mathrm{C}$ & 0.602274 & -1.28628 & -0.94311 \\
\hline $\mathrm{C}$ & -1.94833 & -2.18449 & -1.58962 \\
\hline $\mathrm{C}$ & -0.58165 & -0.65838 & -0.46623 \\
\hline $\mathrm{C}$ & 1.836189 & -0.69949 & -0.51557 \\
\hline $\mathrm{H}$ & -2.95037 & -2.50652 & -1.83464 \\
\hline $\mathrm{C}$ & 1.879839 & 0.383565 & 0.361759 \\
\hline $\mathrm{C}$ & 0.693443 & 1.003063 & 0.858547 \\
\hline $\mathrm{C}$ & 0.666842 & 2.097456 & 1.742469 \\
\hline $\mathrm{C}$ & -0.53498 & 0.468598 & 0.420221 \\
\hline $\mathrm{C}$ & -0.54873 & 2.610564 & 2.141809 \\
\hline $\mathrm{H}$ & 1.597716 & 2.520466 & 2.0973 \\
\hline $\mathrm{H}$ & -0.61071 & 3.450354 & 2.820155 \\
\hline $\mathrm{C}$ & -1.73264 & 2.036886 & 1.663535 \\
\hline $\mathrm{H}$ & -2.69594 & 2.420517 & 1.968584 \\
\hline $\mathrm{N}$ & -1.72753 & 0.993727 & 0.832 \\
\hline $\mathrm{N}$ & 3.169613 & 0.718072 & 0.638192 \\
\hline $\mathrm{N}$ & 3.157589 & -1.00482 & -0.79272 \\
\hline $\mathrm{C}$ & 3.929557 & -0.13311 & -0.04876 \\
\hline $\mathrm{C}$ & 5.392233 & -0.09147 & 0.000677 \\
\hline $\mathrm{C}$ & 8.153206 & 0.040858 & 0.053867 \\
\hline $\mathrm{C}$ & 6.034913 & 1.152628 & 0.23725 \\
\hline $\mathrm{C}$ & 6.165578 & -1.25475 & -0.12769 \\
\hline $\mathrm{C}$ & 7.555337 & -1.21755 & -0.09919 \\
\hline $\mathrm{C}$ & 7.444076 & 1.233263 & 0.228543 \\
\hline $\mathrm{H}$ & 5.658293 & -2.20578 & -0.21637 \\
\hline $\mathrm{H}$ & 9.229993 & 0.093818 & 0.059577 \\
\hline $\mathrm{O}$ & 5.313483 & 2.277759 & 0.463611 \\
\hline $\mathrm{H}$ & 4.382553 & 2.016288 & 0.640874 \\
\hline
\end{tabular}




\begin{tabular}{|c|c|c|c|}
\hline $\mathrm{C}$ & 3.622236 & -1.89198 & -1.86368 \\
\hline $\mathrm{H}$ & 4.634265 & -1.61148 & -2.13802 \\
\hline $\mathrm{H}$ & 3.60892 & -2.93565 & -1.54659 \\
\hline $\mathrm{H}$ & 2.986137 & -1.75805 & -2.73694 \\
\hline $\mathrm{C}$ & 8.175238 & 2.579542 & 0.426785 \\
\hline $\mathrm{C}$ & 7.849135 & 3.159953 & 1.824624 \\
\hline $\mathrm{H}$ & 8.175004 & 2.472276 & 2.610665 \\
\hline $\mathrm{H}$ & 6.782683 & 3.342153 & 1.946035 \\
\hline $\mathrm{H}$ & 8.379948 & 4.106632 & 1.965519 \\
\hline $\mathrm{C}$ & 7.753714 & 3.580981 & -0.67601 \\
\hline $\mathrm{H}$ & 8.010016 & 3.191975 & -1.66601 \\
\hline $\mathrm{H}$ & 8.286135 & 4.527253 & -0.53944 \\
\hline $\mathrm{H}$ & 6.684141 & 3.782795 & -0.65129 \\
\hline $\mathrm{C}$ & 9.70671 & 2.42355 & 0.340269 \\
\hline $\mathrm{H}$ & 10.02701 & 2.034945 & -0.63036 \\
\hline $\mathrm{H}$ & 10.10047 & 1.768408 & 1.121925 \\
\hline $\mathrm{H}$ & 10.17022 & 3.40464 & 0.471176 \\
\hline $\mathrm{C}$ & 8.367714 & -2.52196 & -0.21344 \\
\hline $\mathrm{C}$ & 8.010764 & -3.24728 & -1.53165 \\
\hline $\mathrm{H}$ & 8.233833 & -2.6164 & -2.3966 \\
\hline $\mathrm{H}$ & 6.953166 & -3.51832 & -1.57505 \\
\hline $\mathrm{H}$ & 8.592759 & -4.16903 & -1.62394 \\
\hline $\mathrm{C}$ & 8.028021 & -3.44155 & 0.982892 \\
\hline $\mathrm{H}$ & 8.287673 & -2.9598 & 1.929756 \\
\hline $\mathrm{H}$ & 8.589935 & -4.37784 & 0.912473 \\
\hline $\mathrm{H}$ & 6.964705 & -3.69177 & 1.012523 \\
\hline $\mathrm{C}$ & 9.886317 & -2.26726 & -0.20522 \\
\hline $\mathrm{H}$ & 10.21543 & -1.79145 & 0.722411 \\
\hline $\mathrm{H}$ & 10.19948 & -1.63917 & -1.04378 \\
\hline $\mathrm{H}$ & 10.41379 & -3.2207 & -0.29089 \\
\hline
\end{tabular}

Table S8. Calculated XYZ coordinates of $\left[(\text { bpy })_{2} \mathrm{Ru}^{\mathrm{II}}\left(\text { phen-Im- } \mathrm{PhOH}{ }^{+\bullet}\right)\right]^{3+}$

\begin{tabular}{|c|c|c|c|}
\hline Atom & $\mathrm{X}$ & $\mathrm{Y}$ & $\mathrm{Z}$ \\
\hline $\mathrm{H}$ & -2.09962 & 1.840312 & -4.7092 \\
\hline $\mathrm{C}$ & -2.72192 & 2.018118 & -3.84244 \\
\hline $\mathrm{H}$ & -3.61958 & 3.838003 & -4.57242 \\
\hline $\mathrm{C}$ & -3.56474 & 3.121477 & -3.76283 \\
\hline $\mathrm{N}$ & -3.43163 & 1.288021 & -1.67224 \\
\hline $\mathrm{C}$ & -4.34074 & 3.292465 & -2.62431 \\
\hline $\mathrm{C}$ & -2.68484 & 1.129019 & -2.77887 \\
\hline
\end{tabular}




\begin{tabular}{|c|c|c|c|}
\hline $\mathrm{C}$ & -4.26164 & 2.362558 & -1.58722 \\
\hline $\mathrm{H}$ & -2.04512 & 0.258432 & -2.80458 \\
\hline $\mathrm{C}$ & -5.98148 & 3.469538 & -0.10013 \\
\hline $\mathrm{C}$ & -6.6869 & 3.481012 & 1.095547 \\
\hline $\mathrm{C}$ & -5.05454 & 2.456651 & -0.34705 \\
\hline $\mathrm{C}$ & -6.45213 & 2.473305 & 2.02466 \\
\hline $\mathrm{H}$ & -7.40772 & 4.263574 & 1.29504 \\
\hline $\mathrm{H}$ & -6.97735 & 2.438541 & 2.969687 \\
\hline $\mathrm{C}$ & -5.51981 & 1.491946 & 1.722756 \\
\hline $\mathrm{H}$ & -5.30749 & 0.692743 & 2.417949 \\
\hline $\mathrm{N}$ & -4.83028 & 1.475451 & 0.568522 \\
\hline $\mathrm{Ru}$ & -3.41253 & 0.01266 & 0.014942 \\
\hline $\mathrm{H}$ & -7.19148 & -1.8968 & -3.05301 \\
\hline $\mathrm{C}$ & -6.71105 & -1.99746 & -2.08905 \\
\hline $\mathrm{H}$ & -7.96395 & -3.60013 & -1.37349 \\
\hline $\mathrm{C}$ & -7.13451 & -2.93899 & -1.15723 \\
\hline $\mathrm{N}$ & -5.00491 & -1.24018 & -0.58547 \\
\hline $\mathrm{C}$ & -6.47558 & -3.0201 & 0.062153 \\
\hline $\mathrm{C}$ & -5.64717 & -1.16908 & -1.76464 \\
\hline $\mathrm{C}$ & -5.40889 & -2.16184 & 0.330271 \\
\hline $\mathrm{H}$ & -5.28846 & -0.4256 & -2.46203 \\
\hline $\mathrm{C}$ & -4.88959 & -3.08498 & 2.626627 \\
\hline $\mathrm{C}$ & -4.12554 & -3.02928 & 3.784412 \\
\hline $\mathrm{C}$ & -4.64663 & -2.17978 & 1.593274 \\
\hline $\mathrm{C}$ & -3.12938 & -2.06425 & 3.887305 \\
\hline $\mathrm{H}$ & -4.30697 & -3.7282 & 4.590895 \\
\hline $\mathrm{H}$ & -2.50933 & -1.98011 & 4.769707 \\
\hline $\mathrm{C}$ & -2.93252 & -1.19341 & 2.826058 \\
\hline $\mathrm{H}$ & -2.17089 & -0.42794 & 2.868443 \\
\hline $\mathrm{N}$ & -3.66669 & -1.24201 & 1.700621 \\
\hline $\mathrm{H}$ & -6.15538 & 4.243384 & -0.83366 \\
\hline $\mathrm{H}$ & -5.00115 & 4.143723 & -2.54616 \\
\hline $\mathrm{H}$ & -5.66621 & -3.82979 & 2.530419 \\
\hline $\mathrm{H}$ & -6.79275 & -3.7447 & 0.797939 \\
\hline $\mathrm{H}$ & -1.04038 & -4.15619 & -2.10907 \\
\hline $\mathrm{C}$ & -0.87512 & -3.20191 & -1.62791 \\
\hline $\mathrm{H}$ & 1.245757 & -3.36676 & -1.63903 \\
\hline $\mathrm{C}$ & 0.403021 & -2.74882 & -1.37146 \\
\hline $\mathrm{N}$ & -1.83708 & -1.24862 & -0.63451 \\
\hline $\mathrm{C}$ & 0.582246 & -1.5 & -0.74509 \\
\hline $\mathrm{C}$ & -1.97072 & -2.42172 & -1.26038 \\
\hline
\end{tabular}




\begin{tabular}{|c|c|c|c|}
\hline $\mathrm{C}$ & -0.58516 & -0.78736 & -0.35795 \\
\hline $\mathrm{C}$ & 1.832575 & -0.87279 & -0.41592 \\
\hline $\mathrm{H}$ & -2.98008 & -2.74742 & -1.46746 \\
\hline $\mathrm{C}$ & 1.894706 & 0.316798 & 0.321515 \\
\hline $\mathrm{C}$ & 0.72139 & 1.019158 & 0.746208 \\
\hline $\mathrm{C}$ & 0.727874 & 2.214612 & 1.482513 \\
\hline $\mathrm{C}$ & -0.51586 & 0.458683 & 0.372997 \\
\hline $\mathrm{C}$ & -0.48021 & 2.79874 & 1.805086 \\
\hline $\mathrm{H}$ & 1.665291 & 2.661359 & 1.787063 \\
\hline $\mathrm{H}$ & -0.52544 & 3.720328 & 2.369471 \\
\hline $\mathrm{C}$ & -1.67128 & 2.189669 & 1.398401 \\
\hline $\mathrm{H}$ & -2.62783 & 2.62947 & 1.643961 \\
\hline $\mathrm{N}$ & -1.69987 & 1.047628 & 0.704772 \\
\hline $\mathrm{N}$ & 3.188301 & 0.66133 & 0.517391 \\
\hline $\mathrm{N}$ & 3.138894 & -1.21934 & -0.68968 \\
\hline $\mathrm{C}$ & 3.934516 & -0.27466 & -0.07647 \\
\hline $\mathrm{C}$ & 5.383837 & -0.20646 & -0.02169 \\
\hline $\mathrm{C}$ & 8.173216 & 0.141135 & 0.038437 \\
\hline $\mathrm{C}$ & 5.984559 & 1.078102 & 0.340454 \\
\hline $\mathrm{C}$ & 6.225458 & -1.28143 & -0.24243 \\
\hline $\mathrm{C}$ & 7.625976 & -1.14515 & -0.20746 \\
\hline $\mathrm{C}$ & 7.417295 & 1.258599 & 0.315071 \\
\hline $\mathrm{H}$ & 5.805496 & -2.2595 & -0.41389 \\
\hline $\mathrm{H}$ & 9.245863 & 0.239055 & 0.028922 \\
\hline $\mathrm{O}$ & 5.235792 & 2.075892 & 0.701935 \\
\hline $\mathrm{H}$ & 4.252491 & 1.760699 & 0.744066 \\
\hline $\mathrm{C}$ & 3.572226 & -2.22817 & -1.66573 \\
\hline $\mathrm{H}$ & 4.521597 & -1.92124 & -2.09331 \\
\hline $\mathrm{H}$ & 3.669665 & -3.20952 & -1.20099 \\
\hline $\mathrm{H}$ & 2.84761 & -2.26581 & -2.47493 \\
\hline $\mathrm{C}$ & 8.050123 & 2.626565 & 0.614236 \\
\hline $\mathrm{C}$ & 7.520522 & 3.685773 & -0.38685 \\
\hline $\mathrm{H}$ & 6.447148 & 3.842199 & -0.29456 \\
\hline $\mathrm{H}$ & 8.020706 & 4.638173 & -0.19402 \\
\hline $\mathrm{H}$ & 7.743638 & 3.391283 & -1.41583 \\
\hline $\mathrm{C}$ & 9.583463 & 2.577723 & 0.470833 \\
\hline $\mathrm{H}$ & 9.984143 & 3.573037 & 0.67319 \\
\hline $\mathrm{H}$ & 10.04354 & 1.888993 & 1.18392 \\
\hline $\mathrm{H}$ & 9.893546 & 2.296108 & -0.53888 \\
\hline $\mathrm{C}$ & 7.727144 & 3.053132 & 2.069835 \\
\hline $\mathrm{H}$ & 6.658757 & 3.179925 & 2.234736 \\
\hline
\end{tabular}

\section{S28}




\begin{tabular}{|c|c|c|c|}
\hline H & 8.105638 & 2.315034 & 2.781852 \\
\hline$H$ & 8.220303 & 4.006129 & 2.278278 \\
\hline $\mathrm{C}$ & 8.505332 & -2.37421 & -0.42718 \\
\hline $\mathrm{C}$ & 8.201377 & -2.96857 & -1.82756 \\
\hline $\mathrm{H}$ & 8.834871 & -3.84433 & -1.98855 \\
\hline $\mathrm{H}$ & 7.162344 & -3.28812 & -1.92614 \\
\hline $\mathrm{H}$ & 8.414882 & -2.24289 & -2.61603 \\
\hline $\mathrm{C}$ & 10.00847 & -2.05326 & -0.35026 \\
\hline $\mathrm{H}$ & 10.31507 & -1.33936 & -1.11875 \\
\hline $\mathrm{H}$ & 10.2926 & -1.65775 & 0.628042 \\
\hline $\mathrm{H}$ & 10.5765 & -2.97176 & -0.51083 \\
\hline $\mathrm{C}$ & 8.171935 & -3.42609 & 0.66357 \\
\hline $\mathrm{H}$ & 8.376117 & -3.03187 & 1.661923 \\
\hline $\mathrm{H}$ & 7.128646 & -3.74572 & 0.62758 \\
\hline $\mathrm{H}$ & 8.796764 & -4.30939 & 0.510268 \\
\hline
\end{tabular}

Table S9. Calculated XYZ coordinates of $\left[(\text { bpy })_{2} \mathrm{Ru}^{\mathrm{II}}(\text { phen }-\mathrm{Im}(\mathrm{H})-\mathrm{PhO} \cdot)\right]^{3+}$

\begin{tabular}{|c|c|c|c|}
\hline Atom & $X$ & $\mathrm{Y}$ & $\mathrm{Z}$ \\
\hline $\mathrm{H}$ & -2.35223 & 2.326418 & -4.55403 \\
\hline $\mathrm{C}$ & -2.95551 & 2.391919 & -3.65854 \\
\hline $\mathrm{H}$ & -3.97751 & 4.211061 & -4.20227 \\
\hline $\mathrm{C}$ & -3.85634 & 3.432515 & -3.45995 \\
\hline $\mathrm{N}$ & -3.5547 & 1.438207 & -1.54263 \\
\hline $\mathrm{C}$ & -4.60329 & 3.460481 & -2.29004 \\
\hline $\mathrm{C}$ & -2.83502 & 1.41836 & -2.67822 \\
\hline $\mathrm{C}$ & -4.43946 & 2.451916 & -1.34025 \\
\hline $\mathrm{H}$ & -2.15018 & 0.590884 & -2.79702 \\
\hline $\mathrm{C}$ & -6.15414 & 3.33667 & 0.295044 \\
\hline $\mathrm{C}$ & -6.80562 & 3.214083 & 1.515063 \\
\hline $\mathrm{C}$ & -5.18924 & 2.397713 & -0.07093 \\
\hline $\mathrm{C}$ & -6.47756 & 2.150566 & 2.348938 \\
\hline $\mathrm{H}$ & -7.55588 & 3.937682 & 1.807211 \\
\hline $\mathrm{H}$ & -6.9565 & 2.013327 & 3.309104 \\
\hline $\mathrm{C}$ & -5.51279 & 1.246787 & 1.930002 \\
\hline $\mathrm{H}$ & -5.23049 & 0.407712 & 2.549933 \\
\hline $\mathrm{N}$ & -4.87798 & 1.358192 & 0.749923 \\
\hline $\mathrm{Ru}$ & -3.42424 & 0.014828 & 0.01525 \\
\hline $\mathrm{H}$ & -7.19836 & -1.75531 & -3.13684 \\
\hline $\mathrm{C}$ & -6.6912 & -1.92474 & -2.19651 \\
\hline $\mathrm{H}$ & -7.85226 & -3.6465 & -1.6157 \\
\hline $\mathrm{C}$ & -7.04937 & -2.96998 & -1.35204 \\
\hline
\end{tabular}




\begin{tabular}{|c|c|c|c|}
\hline $\mathrm{N}$ & -4.98506 & -1.23605 & -0.66073 \\
\hline $\mathrm{C}$ & -6.3593 & -3.13583 & -0.15871 \\
\hline $\mathrm{C}$ & -5.65765 & -1.08258 & -1.81482 \\
\hline $\mathrm{C}$ & -5.32694 & -2.25707 & 0.170988 \\
\hline $\mathrm{H}$ & -5.34981 & -0.25958 & -2.44396 \\
\hline $\mathrm{C}$ & -4.72489 & -3.3564 & 2.367758 \\
\hline $\mathrm{C}$ & -3.93202 & -3.37814 & 3.507079 \\
\hline $\mathrm{C}$ & -4.5342 & -2.3592 & 1.410834 \\
\hline $\mathrm{C}$ & -2.9589 & -2.39789 & 3.668438 \\
\hline $\mathrm{H}$ & -4.07352 & -4.14814 & 4.254682 \\
\hline $\mathrm{H}$ & -2.31715 & -2.37271 & 4.538882 \\
\hline $\mathrm{C}$ & -2.81563 & -1.43238 & 2.683372 \\
\hline $\mathrm{H}$ & -2.07416 & -0.65139 & 2.774571 \\
\hline $\mathrm{N}$ & -3.58032 & -1.40336 & 1.577638 \\
\hline $\mathrm{H}$ & -6.39761 & 4.156874 & -0.36448 \\
\hline $\mathrm{H}$ & -5.30553 & 4.263656 & -2.12052 \\
\hline $\mathrm{H}$ & -5.48409 & -4.11193 & 2.227228 \\
\hline $\mathrm{H}$ & -6.62502 & -3.94288 & 0.508446 \\
\hline $\mathrm{H}$ & -0.96032 & -3.83983 & -2.55127 \\
\hline $\mathrm{C}$ & -0.81626 & -2.93784 & -1.97235 \\
\hline $\mathrm{H}$ & 1.305764 & -3.05749 & -1.99581 \\
\hline $\mathrm{C}$ & 0.451188 & -2.48893 & -1.66546 \\
\hline $\mathrm{N}$ & -1.82245 & -1.11951 & -0.78732 \\
\hline $\mathrm{C}$ & 0.602382 & -1.30873 & -0.91194 \\
\hline $\mathrm{C}$ & -1.93005 & -2.2241 & -1.52827 \\
\hline $\mathrm{C}$ & -0.57923 & -0.66 & -0.46445 \\
\hline $\mathrm{C}$ & 1.8394 & -0.6967 & -0.50815 \\
\hline $\mathrm{H}$ & -2.93195 & -2.55144 & -1.76787 \\
\hline $\mathrm{C}$ & 1.861901 & 0.415757 & 0.316539 \\
\hline $\mathrm{C}$ & 0.688263 & 1.075088 & 0.79099 \\
\hline $\mathrm{C}$ & 0.667505 & 2.214407 & 1.61362 \\
\hline $\mathrm{C}$ & -0.53677 & 0.514713 & 0.373749 \\
\hline $\mathrm{C}$ & -0.55316 & 2.737567 & 1.984394 \\
\hline $\mathrm{H}$ & 1.58612 & 2.67756 & 1.949416 \\
\hline $\mathrm{H}$ & -0.61821 & 3.613504 & 2.615256 \\
\hline $\mathrm{C}$ & -1.7305 & 2.125794 & 1.541775 \\
\hline $\mathrm{H}$ & -2.6966 & 2.515638 & 1.829998 \\
\hline $\mathrm{N}$ & -1.73318 & 1.043135 & 0.760668 \\
\hline $\mathrm{N}$ & 3.177388 & 0.714073 & 0.542103 \\
\hline $\mathrm{N}$ & 3.16636 & -1.01789 & -0.7874 \\
\hline $\mathrm{C}$ & 3.970197 & -0.15637 & -0.10832 \\
\hline
\end{tabular}

S30 


\begin{tabular}{|c|c|c|c|}
\hline $\mathrm{C}$ & 5.421483 & -0.11533 & -0.03554 \\
\hline $\mathrm{C}$ & 8.222929 & 0.068415 & 0.04087 \\
\hline $\mathrm{C}$ & 6.059004 & 1.175221 & 0.301672 \\
\hline $\mathrm{C}$ & 6.196428 & -1.24448 & -0.18661 \\
\hline $\mathrm{C}$ & 7.610557 & -1.18948 & -0.13486 \\
\hline $\mathrm{C}$ & 7.526115 & 1.243816 & 0.247573 \\
\hline $\mathrm{H}$ & 5.723062 & -2.20739 & -0.31008 \\
\hline $\mathrm{H}$ & 9.299996 & 0.10689 & 0.034424 \\
\hline $\mathrm{O}$ & 5.363025 & 2.153282 & 0.644151 \\
\hline $\mathrm{H}$ & 3.600334 & 1.518042 & 1.006982 \\
\hline $\mathrm{C}$ & 3.609278 & -1.95871 & -1.82833 \\
\hline $\mathrm{H}$ & 4.603219 & -1.67497 & -2.15844 \\
\hline $\mathrm{H}$ & 3.617485 & -2.98067 & -1.45118 \\
\hline $\mathrm{H}$ & 2.937048 & -1.87184 & -2.67792 \\
\hline $\mathrm{C}$ & 8.233564 & 2.585697 & 0.460785 \\
\hline $\mathrm{C}$ & 7.713349 & 3.623598 & -0.56698 \\
\hline $\mathrm{H}$ & 6.648206 & 3.812017 & -0.44456 \\
\hline $\mathrm{H}$ & 8.249924 & 4.565901 & -0.42811 \\
\hline $\mathrm{H}$ & 7.894655 & 3.281576 & -1.58982 \\
\hline $\mathrm{C}$ & 9.758477 & 2.46557 & 0.274646 \\
\hline $\mathrm{H}$ & 10.20776 & 3.452454 & 0.405535 \\
\hline $\mathrm{H}$ & 10.21414 & 1.799313 & 1.011615 \\
\hline $\mathrm{H}$ & 10.02323 & 2.112123 & -0.72562 \\
\hline $\mathrm{C}$ & 7.968196 & 3.091269 & 1.903254 \\
\hline $\mathrm{H}$ & 6.907393 & 3.260083 & 2.078952 \\
\hline $\mathrm{H}$ & 8.3363 & 2.371421 & 2.639457 \\
\hline $\mathrm{H}$ & 8.502061 & 4.03318 & 2.05701 \\
\hline $\mathrm{C}$ & 8.413036 & -2.48543 & -0.26359 \\
\hline $\mathrm{C}$ & 8.076499 & -3.16563 & -1.61483 \\
\hline $\mathrm{H}$ & 8.663347 & -4.08242 & -1.71265 \\
\hline $\mathrm{H}$ & 7.022371 & -3.43973 & -1.69101 \\
\hline $\mathrm{H}$ & 8.322786 & -2.51151 & -2.45488 \\
\hline $\mathrm{C}$ & 9.932679 & -2.24841 & -0.20515 \\
\hline $\mathrm{H}$ & 10.28025 & -1.61561 & -1.0257 \\
\hline $\mathrm{H}$ & 10.2398 & -1.7938 & 0.739998 \\
\hline $\mathrm{H}$ & 10.44602 & -3.20854 & -0.28978 \\
\hline $\mathrm{C}$ & 8.020918 & -3.43007 & 0.901691 \\
\hline $\mathrm{H}$ & 8.25842 & -2.97824 & 1.867996 \\
\hline $\mathrm{H}$ & 6.957615 & -3.67875 & 0.89311 \\
\hline $\mathrm{H}$ & 8.58206 & -4.36394 & 0.813943 \\
\hline
\end{tabular}




\section{References}

(1) Balfour, W. J. Spectrochim. Acta. A. 1983, 39, 795-800.

(2) Alstrum-Acevedo, J. H.; Brennaman, M. K.; Meyer, T. J. Inorg. Chem. 2005, 44, 6802-6827. 